\title{
Numerical methods for the bidimensional Maxwell-Bloch equations in nonlinear crystals
}

\author{
Antoine Bourgeade ${ }^{a}$ Olivier Saut ${ }^{b}$ \\ ${ }^{a}$ CEA/CESTA, B.P. 2, 33114 Le Barp, France \\ ${ }^{\mathrm{b}}$ MIP, UMR 5640 (CNRS-UPS-INSA), Université Paul Sabatier, 118, route de Narbonne, \\ 31062 Toulouse cedex 4, France
}

\begin{abstract}
Two numerical schemes are developed for solutions of the bidimensional Maxwell-Bloch equations in nonlinear optical crystals. The Maxwell-Bloch model was recently extended [1] to treat anisotropic materials like nonlinear crystals. This semiclassical model seems to be adequate to describe the wave-matter interaction of ultrashort pulses in nonlinear crystals [2] as it is closer to the physics than most macroscopic models. A bidimensional finite-difference-time-domain (FDTD) scheme, adapted from Yee [3], was already developed in [4]. This schemes yields very expensive computations. In this paper, we present two numerical schemes much more efficient with their relative advantages and drawbacks.
\end{abstract}

Key words: Nonlinear optics, Harmonic Generation, Quantum description of light and matter, Nonlinear optical crystal, Numerical schemes.

PACS: 42.65.An, 42.65.Ky, 42.50Ct, 42.70.Mp

\section{Introduction}

Today, laser sources make it possible to produce shorter and shorter, yet powerful, light pulses. Pulses of a few dozen of attoseconds are now obtainable. For such time-length, the classical mathematical models from nonlinear optics are no longer relevant. Because the optical pulses are extremely short, they are beyond the limitations of the slowly varying envelope approximation that is used in the derivation of the nonlinear Schrödinger equation [5,6]. Mathematical models with a dispersive linearity are well known [7]. Adding a quadratic dispersive nonlinearity is a

Email addresses: Antoine.BOURGEADE@cea.fr (Antoine Bourgeade), saut@mip. ups-tlse. fr (Olivier Saut). 
complex task [8,2]. As shown in [1], the Maxwell-Bloch renders any order of the nonlinearity with its dispersive relation.

Furthermore, for practical applications, we would like to study the wave-matter interaction in anisotropic media. We are particularly interested in nonlinear optical crystals with a discrete group of symmetries. Among this large class of crystals, the KDP crystal is of very common use for harmonic generation. This crystal will be used in our experiments.

In [1], we have derived a semiclassical model based on the Maxwell-Bloch equations, adapted to describe the wave-matter interaction in nonlinear crystals. The propagation of an electromagnetic wave is classically described by the full-vector time-dependent Maxwell equations. The modeling of the matter is performed with the Bloch equations, which are derived in the context of quantum mechanics. To obtain the model, we had to find relations between the linear and quadratic optical susceptibilities of the crystal and its quantum structure, which can not be recovered from experimental data. With these relations and careful use of the group of symmetries of the crystal, we were able to postulate the most simple quantum structure adequate to describe the light-matter interaction. The model has three distinct energy levels, one of which is three-fold degenerate.

Then, in [9], we write a discretization scheme when the wave-field depends only on one space variable in the direction of propagation of the laser beam. The electromagnetic field is discretized using the classical Yee scheme [3] with temporal and spatial staggered grids. To obtain a second-order scheme, the points of discretization of the polarization and the density matrix must be chosen carefully. The timederivative of the polarization is computed with the Bloch equations and is replaced in the Maxwell equations by its expression as a function of the density matrix and the electric field. Thus, in order to compute the electric field, at each time step, we have to solve a bloc-diagonal linear system. The Bloch equations are solved using a splitting scheme: the Hamiltonian is divided into the free Hamiltonian and the Hamiltonian resulting from the interaction of the wave-field with the matter.

With this scheme, we compare our model with two macroscopic models based on nonlinear Maxwell equations [2]. In the first one, the polarization is instantaneous, while it takes the linear and quadratic dispersions into account in the second one. We show that the Maxwell-Bloch model renders more physical effects than these macroscopic models. Indeed, with this model, we can see the saturation of the nonlinearity or Raman scattering... Furthermore, the nonlinear polarization is not restricted to its quadratic part as it is in the macroscopic models. Every order of the nonlinearity is computed and takes the dispersion into account.

However, several physical phenomena are still not observable with a one-dimensional model. The diffraction or self-focusing effects can only be seen with a bidimensional study. A first scheme for solutions of the two-dimensional Maxwell-Bloch 
equations was described in [4]. This scheme is a naive extension of the unidimensional FDTD scheme [9]. It discretizes the wave-field described by the pair $(\mathbf{E}, \mathbf{H})$ with a Yee scheme adapted to take the polarization and the density matrix into account. Unfortunately, this scheme yields a large linear system which is to be solved at each time step. It significantly increases its computational cost. Thus, this scheme can only be used for short distances of propagation.

After writing [4], we saw a clear need to develop more efficient schemes for the Maxwell-Bloch equations in order to study numerically realistic physical phenomena. In this article, we present two schemes, which should address this problem. The first scheme uses a pseudospectral method. The time derivatives are still obtained by finite differences, but the spatial derivatives are obtained by mean of a Fourier transform. This scheme is much simpler than the previous one as the use of staggered grids in space for the electric and magnetic fields are no longer mandatory

to ensure a second-order scheme. Because of the wraparound effect of the discrete Fourier transform, absorbing layers have to be added to the computational domain.

The last scheme presented is a FDTD scheme, which should address the main drawback of our first scheme. The three components of the nonlinear polarization are now computed at the same points in space. Moreover, we now describe the wavefield by the pair $(\mathbf{D}, \mathbf{H})$, where $\mathbf{D}$ is the electric induction. The nonlinear polarization term is not explicitly involved in the Maxwell equations.

The outline of this paper is as follows. In the first Section, we present the physical setup and the Maxwell-Bloch model.

In the second Section, we describe the three numerical schemes, their advantages and drawbacks. The first and third ones are FDTD schemes adapted from the Yee scheme [3]. The second one is a pseudospectral scheme adapted from [10].

In the last Section, we perform several numerical experiments to underline some numerical considerations and to study the validity of our model [1].

\section{Physical context}

Let us quickly describe the Maxwell-Bloch model (for a complete derivation of this model, one can see $[11,12])$. In this model, the wave-field is described by the pair $(\mathbf{D}, \mathbf{H}), \mathbf{D}$ being the electric induction and $\mathbf{H}$ the magnetic field, or $(\mathbf{E}, \mathbf{H}), \mathbf{E}$ being the electric field. Its evolution is driven by the Maxwell equations. We assume that the medium does not have any free charge. 
In the $(\mathbf{E}, \mathbf{H})$ formulation, the Maxwell equations are written as

$$
\left\{\begin{array}{l}
\mu_{0} \partial_{t} \mathbf{H}=-\nabla \times \mathbf{E} \\
\varepsilon \partial_{t} \mathbf{E}=\nabla \times \mathbf{H}-\partial_{t} \mathbf{P} \\
\nabla \cdot(\varepsilon \mathbf{E}+\mathbf{P})=0 \\
\nabla \cdot \mathbf{H}=0
\end{array}\right.
$$

We shall denote by $\eta$ the matrix $\varepsilon^{-1}$, the inverse of the static tensor of susceptibility $\varepsilon$.

Remark 1 Let us recall that without any loss of generality in a uniaxial crystal, as shown in [9], we can choose the axis y in order to make four coefficients of the matrices $\varepsilon$ and $\eta$ vanish (namely $\eta_{x y}=\eta_{y x}=\eta_{y z}=\eta_{z y}=0$ ). In the sequel of this paper, we assume that this choice has been made as it simplifies the discretization.

We know that $\mathbf{D}=\varepsilon \mathbf{E}+\mathbf{P}$. Thus, in the $(\mathbf{D}, \mathbf{H})$ formulation, the previous system yields

$$
\left\{\begin{array}{l}
\mu_{0} \partial_{t} \mathbf{H}=-\nabla \times \mathbf{E} \\
\partial_{t} \mathbf{D}=\nabla \times \mathbf{H} \\
\nabla \cdot \mathbf{D}=0 \\
\nabla \cdot \mathbf{H}=0
\end{array}\right.
$$

The two relations $\nabla \cdot \mathbf{D}=0$ and $\nabla \cdot \mathbf{H}=0$ do not play any role in the sequel.

In the Maxwell-Bloch model, the material is statistically described at the quantummechanical level with the density matrix formulation [11]. Each molecule of the crystal is considered as a quantum system with $N$ discrete levels of energy. The density matrix $\rho$ represents in its diagonal terms the various populations in each of the energy levels of the free Hamiltonian. The off-diagonal terms of the density matrix represent the quantum coherences of a set of atomic states.

This density matrix evolves through the Bloch equations:

$$
\partial_{t} \rho_{j k}=-l \omega_{j k} \rho_{j k}+\frac{l}{\hbar}[\mu . \mathbf{E}, \rho]_{j k}, 1 \leq j, k \leq N,
$$

where $\omega_{j k}=\omega_{j}-\omega_{k}, \mu$ is the dipolar matrix characterizing the material at the quantum-mechanical level and $\mathbf{E}$ is the electric field of the incoming laser pulse. We recall that, for two operators $A$ and $B,[A, B] \equiv A B-B A$.

The physical meaning of this model is the following. Each molecule of the crystal is seen as a single atom with one electron independent from the other molecules of 


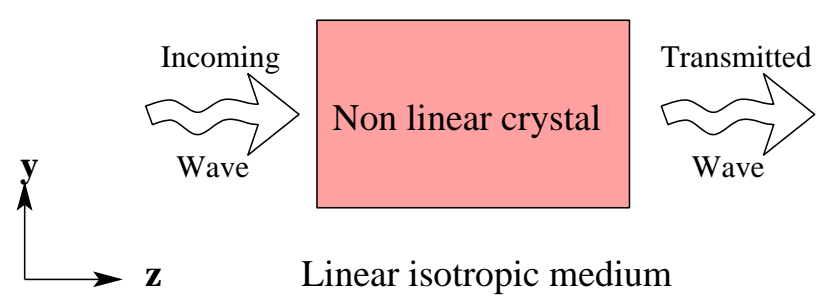

Figure 1. Physical domain.

the crystal. This atom reacts to the wave-field as a quantum dipole. Albeit this oversimplification of the physics involved, this model can be quite accurate to describe the wave-matter interaction.

In this paper, we consider electromagnetic fields depending on two variables in space. One variable $z$ in the direction of propagation of the laser pulse and the other $y$ in a transverse direction. To ease the writing of the boundary conditions, we assume that all interfaces are normal (in fact this is also the experimental setup in order to minimize reflexion and to avoid damaging the optical devices). Fig. 1 presents this setup, the nonlinear optical crystal is surrounded by a linear isotropic material. The linear index of the medium is chosen to minimize the reflexion of the beam at the interfaces with the crystal.

\section{Numerical schemes}

Let us first introduce a notation. The physical quantities $(\mathbf{E}, \mathbf{D}, \mathbf{H}, \mathbf{P}, \rho)$ depend on three variables: the time $t$ and the space variables $y$ (in the transverse direction) and $z$ (in the direction of propagation). The experimental setup is shown in Fig. 1.

For a function $u$ defined on the grid, we write $u_{j, k}^{n}\left(\right.$ or $\left.u\right|_{j, k} ^{n}$ ) for the value of $u$ at the grid point $\left(t_{n}, y_{j}, z_{k}\right)$, where $t_{n}=n \delta t, y_{j}=j \delta y, z_{k}=k \delta z, \delta t$ being the time step, $\delta y$ the space step in the direction $y$ and $\delta z$ the step in direction $z$.

We will denote by $\mathbb{D}^{t}, \mathbb{D}^{y}, \mathbb{D}^{z}$, the central differencing operators in the directions $t$, $y, z$.

For instance, using this notation,

$$
\left(\mathbb{D}^{y} E_{x}\right)_{j+\frac{1}{2}, k}^{n+\frac{1}{2}}=\frac{\left.E_{x}\right|_{j+1, k} ^{n+\frac{1}{2}}-\left.E_{x}\right|_{j, k} ^{n+\frac{1}{2}}}{\delta y} .
$$




\subsection{A first FDTD scheme}

This scheme was deeply described in [4]. For reader's convenience, we shall recall it shortly.

The idea behind [4] was to use the same method as in [9]. That is to say that we use an adapted Yee scheme, where the density matrix is computed at the same points as the electric field $E_{x}$ in space and at the same points as the magnetic field $\mathbf{H}$ in time. This is summarized in Fig. 2.

\subsubsection{Discretization of the Maxwell equations}

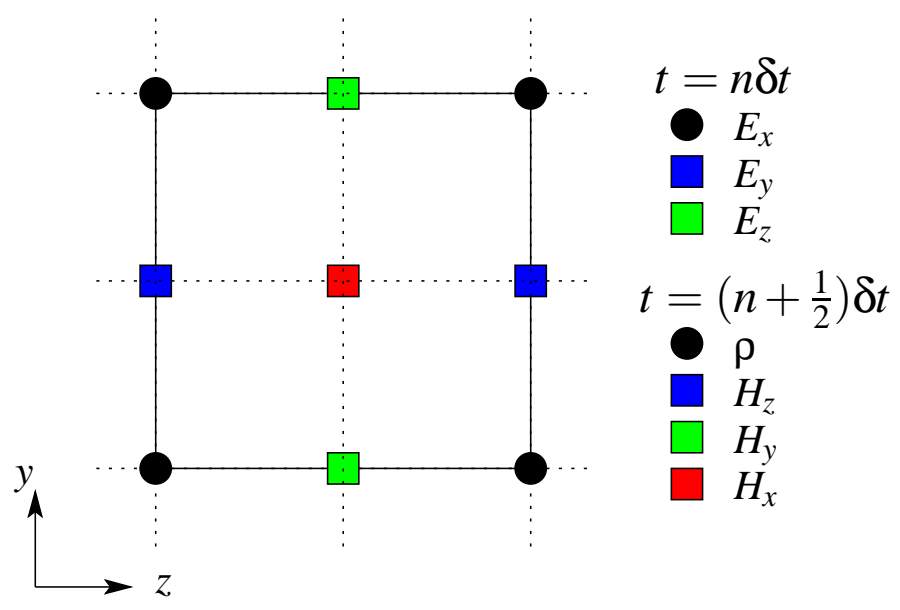

Figure 2. A grid cell of the FDTD scheme [4] for the electromagnetic wave $(\mathbf{E}, \mathbf{H})$ and the density matrix $\rho$.

Using the scheme, we are able to discretize Eq. (1).

For the equations leading the evolution of the magnetic field, we obtain

$$
\begin{aligned}
\mu_{0}\left(\mathbb{D}^{t} H_{x}\right)_{j+\frac{1}{2}, k+\frac{1}{2}}^{n} & =-\left(\mathbb{D}^{y} E_{z}\right)_{j+\frac{1}{2}, k+\frac{1}{2}}^{n}+\left(\mathbb{D}^{z} E_{y}\right)_{j+\frac{1}{2}, k+\frac{1}{2}}^{n} \\
\mu_{0}\left(\mathbb{D}^{t} H_{y}\right)_{j, k+\frac{1}{2}}^{n} & =-\left(\mathbb{D}^{z} E_{x}\right)_{j, k+\frac{1}{2}}^{n} \\
\mu_{0}\left(\mathbb{D}^{t} H_{z}\right)_{j+\frac{1}{2}, k}^{n} & =\left(\mathbb{D}^{y} E_{x}\right)_{j+\frac{1}{2}, k}^{n} .
\end{aligned}
$$


Using the same scheme, we obtain the equations on the electric field $\mathbf{E}$ :

$$
\begin{aligned}
\left(\mathbb{D}^{t} E_{x}\right)_{j, k}^{n+\frac{1}{2}}= & \eta_{x x}\left[\left(\mathbb{D}^{y} H_{z}\right)_{j, k}^{n+\frac{1}{2}}-\left(\mathbb{D}^{z} H_{y}\right)_{j, k}^{n+\frac{1}{2}}\right]-\eta_{x z}\left(\mathbb{D}^{y} H_{x}\right)_{j, k}^{n+\frac{1}{2}} \\
& -\left.\eta_{x x}\left(\partial_{t} P_{x}\right)\right|_{j, k} ^{n+\frac{1}{2}}-\left.\eta_{x z}\left(\partial_{t} P_{z}\right)\right|_{j, k} ^{n+\frac{1}{2}}, \\
\left(\mathbb{D}^{t} E_{y}\right)_{j+\frac{1}{2}, k}^{n+\frac{1}{2}}= & \eta_{y y}\left(\mathbb{D}^{z} H_{x}\right)_{j+\frac{1}{2}, k}^{n+\frac{1}{2}}-\left.\eta_{y y}\left(\partial_{t} P_{y}\right)\right|_{j+\frac{1}{2}, k} ^{n+\frac{1}{2}}, \\
\left(\mathbb{D}^{t} E_{z}\right)_{j, k+\frac{1}{2}}^{n+\frac{1}{2}}= & -\eta_{z z}\left(\mathbb{D}^{y} H_{x}\right)_{j, k+\frac{1}{2}}^{n+\frac{1}{2}}+\eta_{z x}\left[\left.\left(\partial_{y} H_{z}\right)\right|_{j, k+\frac{1}{2}} ^{n+\frac{1}{2}}-\left.\left(\partial_{z} H_{y}\right)\right|_{j, k+\frac{1}{2}} ^{n+\frac{1}{2}}\right] \\
& -\left.\eta_{z x}\left(\partial_{t} P_{x}\right)\right|_{j, k+\frac{1}{2}} ^{n+\frac{1}{2}}-\left.\eta_{z z}\left(\partial_{t} P_{z}\right)\right|_{j, k+\frac{1}{2}} ^{n+\frac{1}{2}},
\end{aligned}
$$

where the two terms $\left.\left(\partial_{y} H_{z}\right)\right|_{j, k+\frac{1}{2}} ^{n+\frac{1}{2}}$ and $\left.\left(\partial_{z} H_{y}\right)\right|_{j, k+\frac{1}{2}} ^{n+\frac{1}{2}}$ in the latter equation can easily be computed with

$$
\left.\left(\partial_{y} H_{z}\right)\right|_{j, k+\frac{1}{2}} ^{n+\frac{1}{2}}=\frac{\left(\mathbb{D}^{y} H_{z}\right)_{j, k+1}^{n+\frac{1}{2}}+\left(\mathbb{D}^{y} H_{z}\right)_{j, k}^{n+\frac{1}{2}}}{2}
$$

and,

$$
\left.\left(\partial_{z} H_{y}\right)\right|_{j, k+\frac{1}{2}} ^{n+\frac{1}{2}}=\frac{\left.H_{y}\right|_{j, k+\frac{3}{2}} ^{n+\frac{1}{2}}-\left.H_{y}\right|_{j, k-\frac{1}{2}} ^{n+\frac{1}{2}}}{2 \delta z} .
$$

The polarization term is still to be determined. For this purpose, we use the continuous expression of $\partial_{t} P$ obtained by deriving the Bloch equations:

$$
\partial_{t} P_{d}=\mathcal{N} \operatorname{tr}(\mu \rho)=\mathcal{N} \operatorname{tr}\left(\mu_{d} \mathrm{R}(\rho)\right)-\frac{l \mathcal{N}}{\hbar} \operatorname{tr}\left(\mu_{d}[V, \rho]\right), d \in\{x, y, z\},
$$

where $V=-E_{x} \mu_{x}-E_{y} \mu_{y}-E_{z} \mu_{z}$. For convenience, we have used the notation

$$
\mathrm{R}(\rho)_{j, k}=-\imath\left(\omega_{j}-\omega_{k}\right) \rho_{j, k}
$$

Then we replace $\partial_{t} \mathbf{P}$ by the expression (8) in the Ampere equations.

For instance, for the first equation of (5), we have to compute the following two terms:

$$
\left.\left(\partial_{t} P_{x}\right)\right|_{j, k} ^{n+\frac{1}{2}}=\mathcal{N} \operatorname{tr}\left(\mu_{x} \mathrm{R}\left(\rho_{j, k}^{n+\frac{1}{2}}\right)\right)-\frac{l \mathcal{N}}{\hbar} \operatorname{tr}\left(\mu_{x}\left[V_{j, k}^{n+\frac{1}{2}}, \rho_{j, k}^{n+\frac{1}{2}}\right]\right),
$$

and,

$$
\left.\left(\partial_{t} P_{z}\right)\right|_{j, k} ^{n+\frac{1}{2}}=\mathcal{N} \operatorname{tr}\left(\mu_{z} \mathrm{R}\left(\rho_{j, k}^{n+\frac{1}{2}}\right)\right)-\frac{l \mathcal{N}}{\hbar} \operatorname{tr}\left(\mu_{z}\left[V_{j, k}^{n+\frac{1}{2}}, \rho_{j, k}^{n+\frac{1}{2}}\right]\right)
$$

With the scheme of Fig. 2 in mind, we now write

$$
V_{j, k}^{n+\frac{1}{2}}=-\left.E_{x}\right|_{j, k} ^{n+\frac{1}{2}} \mu_{x}-\left.E_{y}\right|_{j, k} ^{n+\frac{1}{2}} \mu_{y}-\left.\mu_{z} E_{z}\right|_{j, k} ^{n+\frac{1}{2}} \mu_{y},
$$


where we take

$$
\begin{aligned}
& \left.E_{x}\right|_{j, k} ^{n+\frac{1}{2}} \sim \frac{\left.E_{x}\right|_{j, k} ^{n+1}+\left.E_{x}\right|_{j, k} ^{n}}{2}, \\
& \left.E_{y}\right|_{j, k} ^{n+\frac{1}{2}} \sim \frac{\left.E_{y}\right|_{j+\frac{1}{2}, k} ^{n+1}+\left.E_{y}\right|_{j-\frac{1}{2}, k} ^{n+1}+\left.E_{y}\right|_{j+\frac{1}{2}, k} ^{n}+\left.E_{y}\right|_{j-\frac{1}{2}, k} ^{n}}{4}, \\
& \left.E_{z}\right|_{j, k} ^{n+\frac{1}{2}} \sim \frac{\left.E_{z}\right|_{j, k+\frac{1}{2}} ^{n+1}+\left.E_{z}\right|_{j, k-\frac{1}{2}} ^{n+1}+\left.E_{z}\right|_{j, k+\frac{1}{2}} ^{n}+\left.E_{z}\right|_{j, k-\frac{1}{2}} ^{n}}{4} .
\end{aligned}
$$

Solving the Ampere equations yields a linear system on the electric field $\mathbf{E}$, which could be written as

$$
A \mathbf{E}^{n+1}=F\left(\mathbf{E}^{n}, \mathbf{H}^{n+\frac{1}{2}}, \rho^{n+\frac{1}{2}}\right),
$$

where $A_{j, k}$ is a $\left(N_{y} \times N_{z}\right)^{2}$ matrix and $F$ a linear function with values in $\mathbb{R}^{N_{y} \times N_{z}}$. We have denoted by $N_{y}$ and $N_{z}$ the grid sizes in directions $y$ and $z$ respectively.

This system is not diagonal neither trigonal. It has to be solved using an iterative solver such as GMRES. Because of the resolution of this very large linear system (typical values are $N_{y} \sim 100, N_{z} \sim \frac{100 \ell}{\lambda}$, where $\ell$ is the crystalline depth and $\lambda$ the wavelength of the pulse), the computational cost of this scheme is very high and restricts its use to very short distances of propagation. Furthermore, the development of a parallel algorithm is complex as the iterative solver has to share the computation between the processors. In [13], this was achieved using [14].

It shall be noted that the complexity of this scheme comes from the fact that the three coordinates of the electric field $\mathbf{E}$ are not discretized at the same space points. Then the computation of the polarization term $\partial_{t} \mathbf{P}$ involves taking several averages in space of $\mathbf{E}$ to keep a second order scheme. These space averages yields an implicit scheme of order two in space and time.

\subsubsection{Discretization of the Bloch equations}

Let us recall the method used to discretize the Bloch equations as it was described in [9]. The Bloch equations give

$$
\partial_{t} \rho_{j k}=-\imath \omega_{j k} \rho_{j k}-\frac{l}{\hbar}[V, \rho]_{j k}, 1 \leq j, k \leq N,
$$

where $V=-E_{x} \mu_{x}-E_{y} \mu_{y}-E_{z} \mu_{z}$.

This equation is divided in two parts. The first one

$$
\partial_{t} \rho_{j k}=-l \omega_{j k} \rho_{j k}, 1 \leq j, k \leq N
$$


can easily be rewritten as a diagonal system. The solving operator of this equation is denoted by $S_{\mathcal{H}_{0}}$.

The second one

$$
\partial_{t} \rho_{j k}=-\frac{l}{\hbar}[V, \rho]_{j k}, 1 \leq j, k \leq N,
$$

is solved thanks to the exact solution of this equation

$$
\rho(t)=\exp \left(-\frac{l}{\hbar} \int_{0}^{t} V(s) d s\right) \rho(0) \exp \left(\frac{l}{\hbar} \int_{0}^{t} V(s) d s\right) .
$$

The integral is computed by the formula

$$
\int_{0}^{t} V(s) d s \sim t V\left(\frac{t}{2}\right)
$$

The exponential of a matrix $M$ is obtained with

$$
\exp M \sim\left(\mathrm{I}-\frac{1}{2} M\right)^{-1}\left(\mathrm{I}+\frac{1}{2} M\right)
$$

We use this approximation to ensure that the discretized density matrix $\rho^{n+\frac{1}{2}}$ keeps the same algebraic properties as the continuous one (see $[15,16]): \rho$ should be hermitian, with a unity trace. With some other discretizations (as [17]), some diagonal terms of the density matrix could take negative values and the trace could be greater than 1 .

The solving operator of Eq. (14) will be denoted by $S_{\mathcal{V}}$.

Using a Strang splitting method, we have a second-order scheme for the Bloch equations. The density matrix is computed by

$$
\rho_{j, k}^{n+\frac{1}{2}}=S_{\mathcal{H}_{0}}^{\frac{1}{2}} S_{\mathcal{V}} S_{\mathcal{H}_{0}}^{\frac{1}{2}} \rho_{j, k}^{n-\frac{1}{2}}
$$

Let us note that, at each step, the potential $V$ has to be computed as

$$
V_{j, k}^{n}=-\left.E_{x}\right|_{j, k} ^{n} \mu_{x}-\frac{\left.E_{y}\right|_{j+\frac{1}{2}, k} ^{n}+\left.E_{y}\right|_{j-\frac{1}{2}, k} ^{n}}{2} \mu_{y}-\frac{\left.E_{z}\right|_{j, k+\frac{1}{2}} ^{n}+\left.E_{z}\right|_{j, k-\frac{1}{2}} ^{n}}{2} \mu_{z} .
$$

\subsection{Pseudospectral scheme}

This scheme was very briefly announced in [18]. The main goal of this scheme is to lighten the computational burden of the resolution of the bidimensional MaxwellBloch equations with the scheme described in Sec. 3.1. We adapt an idea from [10]. 
The complexity of [4] comes from the fact that, to keep a second-order scheme, we have to use staggered grids for the three components of the electric field. This leads to a very large linear system to solve at each time step in order to obtain the electric field.

Hence, we have tried to avoid the use of spatial staggered grids. As in [10], we shall use Fourier transforms to compute the spatial derivatives of the electric field $\mathbf{E}$ and magnetic field $\mathbf{H}$ with a spectral order of accuracy. For a function $f$ of the space variable $x \in \mathbb{R}^{3}$, its derivative in the direction $d$ is given by

$$
\partial_{d} f(x)=\left[\overline{\mathcal{F}}\left(-\imath \xi_{d} \mathcal{F}(f)\right)\right](x)
$$

where $\xi_{d}$ is the spectral variable in the direction $d$. The discrete Fourier transform is achieved by using the fast-Fourier-transform (FFT) algorithm. We denote by $\partial_{d}^{F}$ the discrete derivative in direction $d$.

The temporal grid is still staggered because central differencing is used for time stepping.

The Bloch equations are solved using the method described in Sec. 3.1.2. The only difference is that the electric field is discretized in the same points in space as the density matrix so there is no need for the approximation (19).

\subsubsection{Discretization of the Maxwell equations}

To compute the polarization term $\partial_{t} \mathbf{P}$ involved in the Ampere equations, we use the same method as in Section 3.1 with the Bloch equations. However, the density matrix and the components of the electric field are now approximated on the same grid points in space (see Fig. 3).

For the equations leading the evolution of the magnetic field, we obtain

$$
\begin{aligned}
& \mu_{0}\left(\mathbb{D}^{t} H_{x}\right)_{j, k}^{n}=-\left(\partial_{y}^{F} E_{z}\right)_{j, k}^{n}+\left(\partial_{z}^{F} E_{y}\right)_{j, k}^{n}, \\
& \mu_{0}\left(\mathbb{D}^{t} H_{y}\right)_{j, k}^{n}=-\left(\partial_{z}^{F} E_{x}\right)_{j, k}^{n}, \\
& \mu_{0}\left(\mathbb{D}^{t} H_{z}\right)_{j, k}^{n}=\left(\partial_{y}^{F} E_{x}\right)_{j, k}^{n} .
\end{aligned}
$$


Using the same scheme, we obtain the equations on the electric field $\mathbf{E}$ :

$$
\begin{aligned}
\left(\mathbb{D}^{t} E_{x}\right)_{j, k}^{n+\frac{1}{2}}= & \eta_{x x}\left[\left(\partial_{y}^{F} H_{z}\right)_{j, k}^{n+\frac{1}{2}}-\left(\partial_{z}^{F} H_{y}\right)_{j, k}^{n+\frac{1}{2}}\right]-\eta_{x z}\left(\partial_{y}^{F} H_{x}\right)_{j, k}^{n+\frac{1}{2}} \\
& -\left.\eta_{x x}\left(\partial_{t} P_{x}\right)\right|_{j, k} ^{n+\frac{1}{2}}-\left.\eta_{x z}\left(\partial_{t} P_{z}\right)\right|_{j, k} ^{n+\frac{1}{2}}, \\
\left(\mathbb{D}^{t} E_{y}\right)_{j, k}^{n+\frac{1}{2}}= & \eta_{y y}\left(\partial_{z}^{F} H_{x}\right)_{j, k}^{n+\frac{1}{2}}-\left.\eta_{y y}\left(\partial_{t} P_{y}\right)\right|_{j, k} ^{n+\frac{1}{2}}, \\
\left(\mathbb{D}^{t} E_{z}\right)_{j, k}^{n+\frac{1}{2}}= & -\eta_{z z}\left(\partial_{y}^{F} H_{x}\right)_{j, k}^{n+\frac{1}{2}}+\eta_{z x}\left[\left.\left(\partial_{y}^{F} H_{z}\right)\right|_{j, k} ^{n+\frac{1}{2}}-\left.\left(\partial_{z}^{F} H_{y}\right)\right|_{j, k} ^{n+\frac{1}{2}}\right] \\
& -\left.\eta_{z x}\left(\partial_{t} P_{x}\right)\right|_{j, k} ^{n+\frac{1}{2}}-\left.\eta_{z z}\left(\partial_{t} P_{z}\right)\right|_{j, k} ^{n+\frac{1}{2}} .
\end{aligned}
$$

The polarization terms are computed with Eq. (8):

$$
\left.\left(\partial_{t} P_{d}\right)\right|_{j, k} ^{n+\frac{1}{2}}=\mathcal{N} \operatorname{tr}\left(\mu_{d} \mathrm{R}\left(\rho_{j, k}^{n+\frac{1}{2}}\right)\right)-\frac{l \mathcal{N}}{\hbar} \operatorname{tr}\left(\mu_{d}\left[V_{j, k}^{n+\frac{1}{2}}, \rho_{j, k}^{n+\frac{1}{2}}\right]\right), d \in\{x, y, z\}
$$

so $\rho_{j, k}^{n+\frac{1}{2}}$ and $V_{j, k}^{n+\frac{1}{2}}$ are to be approximated to determine these terms.

We have a second order approximation for $V_{j, k}^{n+\frac{1}{2}}$ with

$$
V_{j, k}^{n+\frac{1}{2}}=-\frac{\left.E_{x}\right|_{j, k} ^{n+1}+\left.E_{x}\right|_{j, k} ^{n}}{2} \mu_{x}-\frac{\left.E_{y}\right|_{j, k} ^{n+1}+\left.E_{y}\right|_{j, k} ^{n}}{2} \mu_{y}-\frac{\left.E_{z}\right|_{j, k} ^{n+1}+\left.E_{z}\right|_{j, k} ^{n}}{2} \mu_{z} .
$$

The density matrix $\rho^{n+\frac{1}{2}}$ is obtained thanks to the Bloch equations. We replace $\mathrm{V}$ by its value (24) in equation (23), then $\left.\left(\partial_{t} P_{d}\right)\right|_{j, k} ^{n+\frac{1}{2}}$ from Eq. (23) is injected into equations (22).

Let us write the corresponding equation for $\left.E_{y}\right|_{j, k} ^{n+1}$. From the second equation of (22) and Eq. (23), (24), we have

$$
\begin{aligned}
\frac{\left.E_{y}\right|_{j, k} ^{n+1}-\left.E_{y}\right|_{j, k} ^{n}}{\delta t}= & \eta_{y y}\left(\partial_{z}^{F} H_{x}\right)_{j, k}^{n+\frac{1}{2}}-\eta_{y y} \mathcal{N} \operatorname{tr}\left(\mu_{y} \mathrm{R}\left(\rho_{j, k}^{n+\frac{1}{2}}\right)\right) \\
& -\eta_{y y} \frac{l \mathcal{N}}{\hbar} \frac{\left.E_{x}\right|_{j, k} ^{n+1}+\left.E_{x}\right|_{j, k} ^{n}}{2} \operatorname{tr}\left(\mu_{y}\left[\mu_{x}, \rho_{j, k}^{n+\frac{1}{2}}\right]\right) \\
& -\eta_{y y} \frac{l \mathcal{N}}{\hbar} \frac{\left.E_{z}\right|_{j, k} ^{n+1}+\left.E_{z}\right|_{j, k} ^{n}}{2} \operatorname{tr}\left(\mu_{y}\left[\mu_{z}, \rho_{j, k}^{n+\frac{1}{2}}\right]\right) .
\end{aligned}
$$

The component $\left.E_{y}\right|_{j, k} ^{n+1}$ is computed at each time step with 


$$
\begin{aligned}
\left.E_{y}\right|_{j, k} ^{n+1}= & -\eta_{y y} \frac{i \delta t \mathcal{N}}{2 \hbar}\left(\left.\operatorname{tr}\left(\mu_{y}\left[\mu_{x}, \rho_{j, k}^{n+\frac{1}{2}}\right]\right) E_{x}\right|_{j, k} ^{n+1}+\left.\operatorname{tr}\left(\mu_{y}\left[\mu_{z}, \rho_{j, k}^{n+\frac{1}{2}}\right]\right) E_{z}\right|_{j, k} ^{n+1}\right) \\
& -\eta_{y y} \frac{l \delta t \mathcal{N}}{2 \hbar}\left(\left.\operatorname{tr}\left(\mu_{y}\left[\mu_{x}, \rho_{j, k}^{n+\frac{1}{2}}\right]\right) E_{x}\right|_{j, k} ^{n}+\left.\operatorname{tr}\left(\mu_{y}\left[\mu_{z}, \rho_{j, k}^{n+\frac{1}{2}}\right]\right) E_{z}\right|_{j, k} ^{n}\right) \\
& +\left.E_{y}\right|_{j, k} ^{n}+\delta t \eta_{y y}\left(\partial_{z}^{F} H_{x}\right)_{j, k}^{n+\frac{1}{2}}-\delta t \eta_{y y} \mathcal{N} \operatorname{tr}\left(\mu_{y} \mathrm{R}\left(\rho_{j, k}^{n+\frac{1}{2}}\right)\right) .
\end{aligned}
$$

Remark 2 We have used several relations to get Eq. (26). We know that $\operatorname{tr}(A[A, B])=$ $0, \forall A, B\left(\right.$ then $\left.\operatorname{tr}\left(\mu_{y}\left[\mu_{y}, \rho_{j, k}^{n+\frac{1}{2}}\right]\right)=0\right)$. As the matrices $\mu$ and $\rho$ are Hermitian, we also have $\operatorname{tr}\left(\mu_{d_{1}}\left[\mu_{d_{2}}, \rho_{j, k}^{n+\frac{1}{2}}\right]\right) \in \imath \mathbb{R}$ and $\operatorname{tr}\left(\mu_{d} \mathrm{R}\left(\rho_{j, k}^{n+\frac{1}{2}}\right)\right) \in \mathbb{R}$.

The first line of (26) contains the quantities to compute at time $t_{n+1}$, the two remaining lines involves quantities computed at the previous iteration (for $t \leq t_{n+\frac{1}{2}}$ ). In order to simplify the equations, we introduce the following notations

$$
\mathbb{T}_{d_{1} d_{2}}(\rho)=\frac{l \mathcal{N}}{\hbar} \operatorname{tr}\left(\mu_{d_{1}}\left[\mu_{d_{2}}, \rho\right]\right), d_{1}, d_{2} \in\{x, y, z\}
$$

Eq. (26) may be rewritten as

$$
\begin{aligned}
\left.E_{y}\right|_{j, k} ^{n+1}= & -\eta_{y y} \frac{\delta t}{2}\left(\left.\mathbb{T}_{y x}\left(\rho^{n+\frac{1}{2}}\right) E_{x}\right|_{j, k} ^{n+1}+\left.\mathbb{T}_{y z}\left(\rho_{j, k}^{n+\frac{1}{2}}\right) E_{z}\right|_{j, k} ^{n+1}\right) \\
& -\eta_{y y} \frac{\delta t}{2}\left(\left.\mathbb{T}_{y x}\left(\rho_{j, k}^{n+\frac{1}{2}}\right) E_{x}\right|_{j, k} ^{n}+\left.\mathbb{T}_{y z}\left(\rho_{j, k}^{n+\frac{1}{2}}\right) E_{z}\right|_{j, k} ^{n}\right) \\
& +\left.E_{y}\right|_{j, k} ^{n}+\delta t \eta_{y y}\left(\partial_{z}^{F} H_{x}\right)_{j, k}^{n+\frac{1}{2}}-\delta t \eta_{y y} \mathcal{N} \operatorname{tr}\left(\mu_{y} \mathrm{R}\left(\rho_{j, k}^{n+\frac{1}{2}}\right)\right) .
\end{aligned}
$$

The two remaining components are obtained using the same method: 


$$
\begin{aligned}
\left.E_{x}\right|_{j, k} ^{n+1}= & -\eta_{x x} \frac{\delta t}{2}\left(\left.\mathbb{T}_{x y}\left(\rho_{j, k}^{n+\frac{1}{2}}\right) E_{y}\right|_{j, k} ^{n+1}+\left.\mathbb{T}_{x z}\left(\rho_{j, k}^{n+\frac{1}{2}}\right) E_{z}\right|_{j, k} ^{n+1}\right) \\
& -\eta_{x z} \frac{\delta t}{2}\left(\left.\mathbb{T}_{z x}\left(\rho_{j, k}^{n+\frac{1}{2}}\right) E_{x}\right|_{j, k} ^{n+1}+\left.\mathbb{T}_{z y}\left(\rho_{j, k}^{n+\frac{1}{2}}\right) E_{y}\right|_{j, k} ^{n+1}\right) \\
& -\eta_{x x} \frac{\delta t}{2}\left(\left.\mathbb{T}_{x y}\left(\rho_{j, k}^{n+\frac{1}{2}}\right) E_{y}\right|_{j, k} ^{n}+\left.\mathbb{T}_{x z}\left(\rho_{j, k}^{n+\frac{1}{2}}\right) E_{z}\right|_{j, k} ^{n}\right) \\
& -\eta_{x z} \frac{\delta t}{2}\left(\left.\mathbb{T}_{z x}\left(\rho_{j, k}^{n+\frac{1}{2}}\right) E_{x}\right|_{j, k} ^{n}+\left.\mathbb{T}_{z y}\left(\rho_{j, k}^{n+\frac{1}{2}}\right) E_{y}\right|_{j, k} ^{n}\right) \\
& +\left.E_{x}\right|_{j, k} ^{n}+\delta t \eta_{x x}\left[\left(\partial_{y}^{F} H_{z}\right)_{j, k}^{n+\frac{1}{2}}-\left(\partial_{z}^{F} H_{y}\right)_{j, k}^{n+\frac{1}{2}}\right] \\
& -\delta t\left(\eta_{x z}\left(\partial_{y}^{F} H_{x}\right)_{j, k}^{n+\frac{1}{2}}-\eta_{x x} \mathcal{N} \operatorname{tr}\left(\mu_{x} \mathrm{R}\left(\rho_{j, k}^{n+\frac{1}{2}}\right)\right)-\eta_{x z} \mathcal{N} \operatorname{tr}\left(\mu_{z} \mathrm{R}\left(\rho_{j, k}^{n+\frac{1}{2}}\right)\right)\right),
\end{aligned}
$$

and

$$
\begin{aligned}
\left.E_{z}\right|_{j, k} ^{n+1}= & -\eta_{z x} \frac{\delta t}{2}\left(\left.\mathbb{T}_{x y}\left(\rho_{j, k}^{n+\frac{1}{2}}\right) E_{y}\right|_{j, k} ^{n+1}+\left.\mathbb{T}_{x z}\left(\rho_{j, k}^{n+\frac{1}{2}}\right) E_{z}\right|_{j, k} ^{n+1}\right) \\
& -\eta_{z z} \frac{\delta t}{2}\left(\left.\mathbb{T}_{z x}\left(\rho_{j, k}^{n+\frac{1}{2}}\right) E_{x}\right|_{j, k} ^{n+1}+\left.\mathbb{T}_{z y}\left(\rho_{j, k}^{n+\frac{1}{2}}\right) E_{y}\right|_{j, k} ^{n+1}\right) \\
& -\eta_{z x} \frac{\delta t}{2}\left(\left.\mathbb{T}_{x y}\left(\rho_{j, k}^{n+\frac{1}{2}}\right) E_{y}\right|_{j, k} ^{n}+\left.\mathbb{T}_{x z}\left(\rho_{j, k}^{n+\frac{1}{2}}\right) E_{z}\right|_{j, k} ^{n}\right) \\
& -\eta_{z z} \frac{\delta t}{2}\left(\left.\mathbb{T}_{z x}\left(\rho_{j, k}^{n+\frac{1}{2}}\right) E_{x}\right|_{j, k} ^{n}+\left.\mathbb{T}_{z y}\left(\rho_{j, k}^{n+\frac{1}{2}}\right) E_{y}\right|_{j, k} ^{n}\right) \\
& +\left.E_{z}\right|_{j, k} ^{n}+\delta t \eta_{z x}\left[\left(\partial_{y}^{F} H_{z}\right)_{j, k}^{n+\frac{1}{2}}-\left(\partial_{z}^{F} H_{y}\right)_{j, k}^{n+\frac{1}{2}}\right] \\
& -\delta t\left(\eta_{z z}\left(\partial_{y}^{F} H_{x}\right)_{j, k}^{n+\frac{1}{2}}-\eta_{z x} \mathcal{N} \operatorname{tr}\left(\mu_{x} \mathrm{R}\left(\rho_{j, k}^{n+\frac{1}{2}}\right)\right)-\eta_{z z} \mathcal{N} \operatorname{tr}\left(\mu_{z} \mathrm{R}\left(\rho_{j, k}^{n+\frac{1}{2}}\right)\right)\right) .
\end{aligned}
$$

Collecting the above equations, we may rewrite the equations (22) as a single linear system

$$
A_{j, k} \mathbf{E}_{j, k}^{n+1}=F_{j, k}\left(\mathbf{E}_{j, k}^{n}, \rho_{j, k}^{n+\frac{1}{2}}, \partial_{y}^{F} \mathbf{H}_{j, k}^{n+\frac{1}{2}}, \partial_{z}^{F} \mathbf{H}_{j, k}^{n+\frac{1}{2}}\right), \forall(j, k),
$$

where $A_{j, k}$ is a $3 \times 3$ matrix and $F_{j, k}$ a linear function.

Thus, the resolution of the Ampere equations on the whole domain, yields a bloc diagonal linear system (blocs are the $3 \times 3$ sub-matrices given by the equation (28) for each point $(j, k)$ of the domain). This system is easily solvable by a direct method.

\subsubsection{Boundary conditions}

The consequence of using a discrete Fourier transform (FFT) for the spatial derivatives is to periodize the domain in both directions. The domain could be considered 


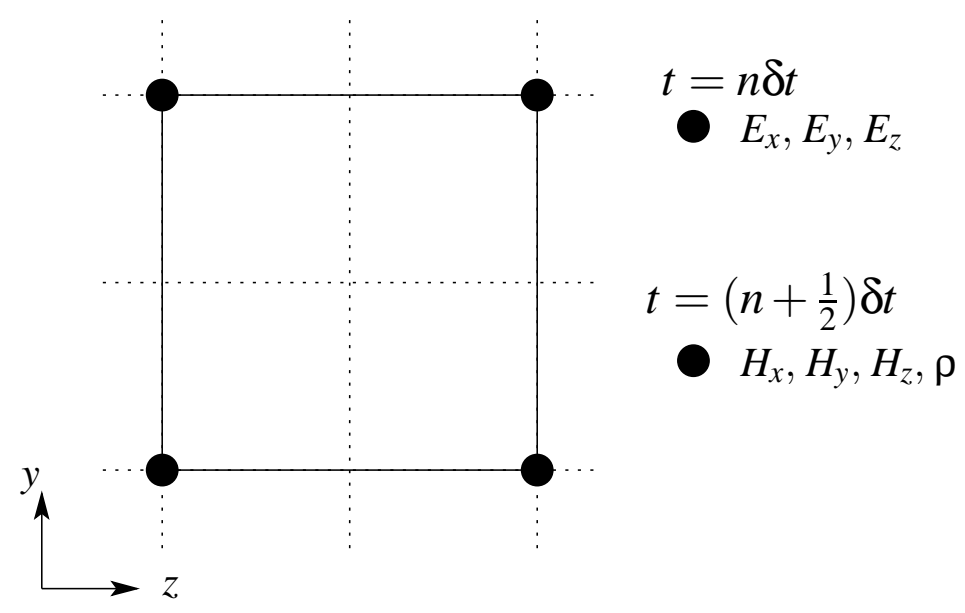

Figure 3. Pseudospectral scheme for the electromagnetic wave $(\mathbf{E}, \mathbf{H})$ and the density matrix $\rho$.

as a tore. The pulses, which, physically, should leave the domain, now reenters the computational domain and may interfere with the pulses still in propagation. This is called the wraparound effect.

To counter the wraparound effect due to the FFT, we use perfectly matched layers (PML) boundary conditions [19]. We shall now describe the use of these conditions in our particular case.

In the absorbing layers, the electric field $\mathbf{E}$ and magnetic field $\mathbf{H}$ in the $x$-direction are decomposed as

$$
\begin{aligned}
& E_{x}=E_{x y}+E_{x z}, \\
& H_{x}=H_{x y}+H_{x z} .
\end{aligned}
$$

Then the electric field is driven in the PML cells by

$$
\begin{gathered}
\partial_{t} E_{x y}+\sigma_{y} E_{x y}=-\mu_{0} c^{2} \partial_{y} H_{z}, \\
\partial_{t} E_{x z}+\sigma_{z} E_{x z}=\mu_{0} c^{2} \partial_{z} H_{y}, \\
\partial_{t} E_{y}+\sigma_{z} E_{y}=\mu_{0} c^{2} \partial_{z} H_{x}, \\
\partial_{t} E_{z}+\sigma_{y} E_{z}=-\mu_{0} c^{2} \partial_{y} H_{x},
\end{gathered}
$$

and the magnetic field by

$$
\begin{gathered}
\partial_{t} H_{x z}+\sigma_{z}^{*} H_{x z}=\mu_{0}^{-1} \partial_{z} E_{y}, \\
\partial_{t} H_{x z}+\sigma_{y}^{*} H_{x z}=-\mu_{0}^{-1} \partial_{y} E_{z}, \\
\partial_{t} H_{y}+\sigma_{z}^{*} H_{y}=-\mu_{0}^{-1} \partial_{z} E_{x}, \\
\partial_{t} H_{z y}+\sigma_{y}^{*} H_{z y}=\mu_{0}^{-1} \partial_{y} E_{x} .
\end{gathered}
$$


These equations are discretized using an exponential time step as shown in [7].

When $\sigma_{y}=\sigma_{z}=\sigma_{y}^{*}=\sigma_{z}^{*}=0$, we get the Maxwell equations in a linear medium. In the absorbing layers, we use a quadratic or cubic profile with 10 PML cells. In Fig. 4, we have represented the areas where the PML coefficients $\sigma$ and $\sigma^{*}$ are not vanishing. For instance, we took at the entry of the domain

$$
\sigma_{z}(k)=\sigma_{m z}\left(1-\frac{k}{n_{z}}\right)^{3}
$$

where $k$ is the z-coordinate of the point considered, $n_{z}$ the number of PML cells in this direction and $\sigma_{m z}=114.9 \frac{c}{n_{z} \delta z}$. We obtain $\sigma_{z}$ at the exit by periodicity. For the PML cells in direction $y$, we use the same formula after replacing $\delta z$ by $\delta y, n_{z}$ by $n_{y}$.

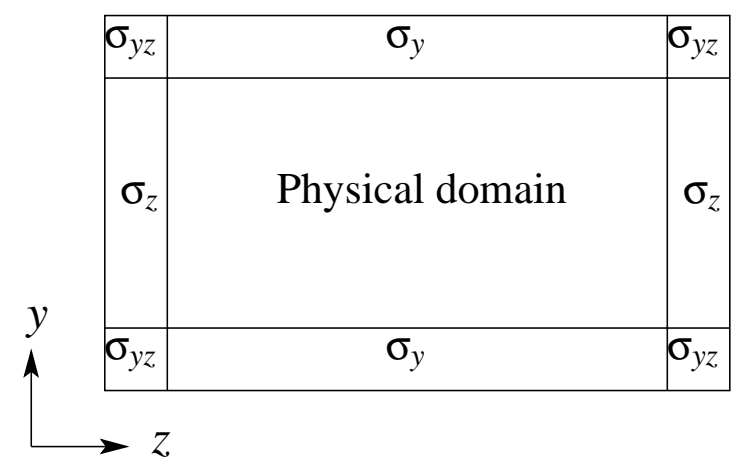

Figure 4. Non-vanishing PML coefficients in the absorbing layers around the physical domain.

In contrast with the previous scheme, the incoming wave-field can not be introduced within the boundary conditions. So the incoming pulse should be put in the domain as initial datum. The linear medium before the crystal has to be large enough to contain entirely this datum (this prevents the study of sinusoidal waves for instance).

\subsubsection{Parallelization}

In the Bloch equations (3), the space variables $y$ and $z$ act as parameters. These equations do not involve any spatial derivatives. They can be solved at each point in space independently from each other. The sharing of the resolution between several processing units is straightforward. The computational domain is divided as described in Fig. 5. In fact, as shown in [13], a quasi linear speed-up with the number of CPU could be observed.

The development of parallel algorithms for the Yee scheme is also well known (see [7]). In our case, the computation of the polarization term should also be shared 


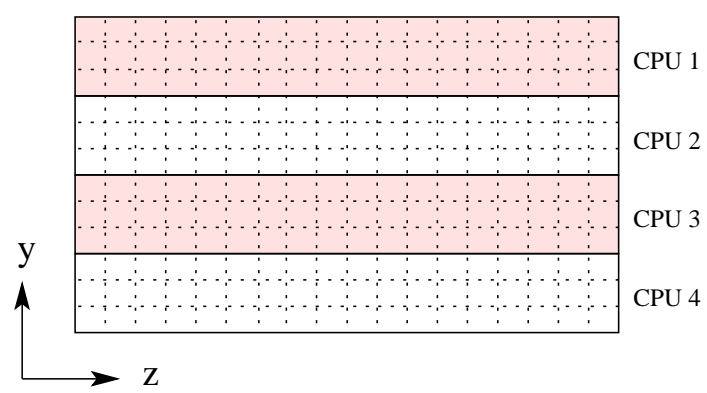

Figure 5. Load sharing between four processors.

between the CPU. With the first scheme of Sec. 3.1, this yields a severe decrease of the efficiency of the parallel method [13].

With the pseudospectral scheme, the polarization term is computed on the same points in space as the wave-field and the density matrix. Their computations are purely local to a given CPU. The only process requiring exchanges between the processing units is the computation of the spatial derivatives in the $y$-direction. With a parallel algorithm for the FFT, this scales well with the number of CPU.

\subsubsection{Conclusion}

In this scheme, we have used central differencing and central averaging in time. The spatial derivatives are computed on the whole domain with a FFT algorithm.

Proposition 3 The PSTD scheme is of second order in time and space.

From [10], we could get the stability criterion for the linear scheme (i.e. without the polarization term)

$$
c \delta t \sqrt{\frac{1}{\delta z^{2}}+\frac{1}{\delta y^{2}}}<\frac{2}{\pi},
$$

where $c$ is the speed of light in the medium, $\delta y$ and $\delta z$ the two space steps.

Furthermore, in contrast with FDTD schemes, this scheme do not introduce numerical dispersion through the approximation of the spatial derivatives. This is quite interesting when studying the propagation of ultrashort pulses in media with dispersive nonlinearities.

\subsection{Second FDTD scheme using a centered nonlinearity}

The main drawback of the scheme 3.1 can also be alleviated without using a spectral method. In this section, we shall present a new FDTD scheme. We will describe the wave field with the pair $(\mathbf{D}, \mathbf{H})$ instead of the pair $(\mathbf{E}, \mathbf{H})$. 


\subsubsection{Discretization of the Maxwell equations}

In the bidimensional case, in the $(\mathbf{D}, \mathbf{H})$ formulation, the Maxwell equations become

$$
\left\{\begin{aligned}
\partial_{t} D_{x} & =\partial_{y} H_{z}-\partial_{z} H_{y} \\
\partial_{t} D_{y} & =\partial_{z} H_{x} \\
\partial_{t} D_{z} & =-\partial_{y} H_{x} \\
\mu_{0} \partial_{t} H_{x} & =-\partial_{y} E_{z}+\partial_{z} E_{y} \\
\mu_{0} \partial_{t} H_{y} & =-\partial_{z} E_{x} \\
\mu_{0} \partial_{t} H_{z} & =\partial_{y} E_{x}
\end{aligned}\right.
$$

The Maxwell equations on $(\mathbf{D}, \mathbf{H})$ do not involve explicitly the polarization $\mathbf{P}$. However, the polarization is still necessary to compute $\mathbf{D}$ from $\mathbf{E}$ or $\mathbf{E}$ from $\mathbf{D}$.

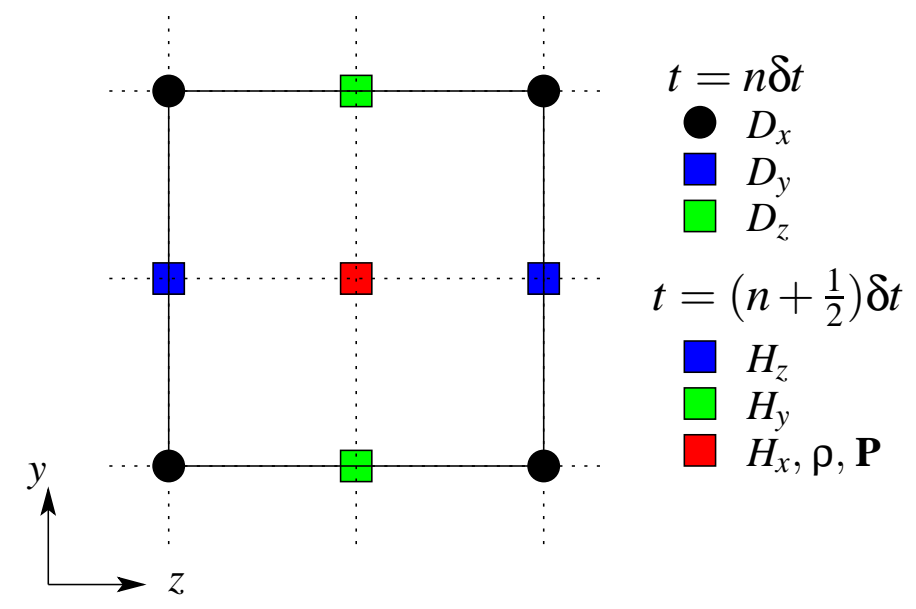

Figure 6. FDTD scheme with centered nonlinearity for the electromagnetic wave $(\mathbf{D}, \mathbf{H})$, the polarization $\mathbf{P}$ and the density matrix $\rho$.

With the scheme of Fig. 6, we obtain the following set of equations

$$
\begin{aligned}
\mu_{0}\left(\mathbb{D}^{t} H_{x}\right)_{j+\frac{1}{2}, k+\frac{1}{2}}^{n} & =-\left(\mathbb{D}^{y} E_{z}\right)_{j+\frac{1}{2}, k+\frac{1}{2}}^{n}+\left(\mathbb{D}^{z} E_{y}\right)_{j+\frac{1}{2}, k+\frac{1}{2}}^{n} \\
\mu_{0}\left(\mathbb{D}^{t} H_{y}\right)_{j, k+\frac{1}{2}}^{n} & =-\left(\mathbb{D}^{z} E_{x}\right)_{j, k+\frac{1}{2}}^{n}, \\
\mu_{0}\left(\mathbb{D}^{t} H_{z}\right)_{j+\frac{1}{2}, k}^{n} & =\left(\mathbb{D}^{y} E_{x}\right)_{j+\frac{1}{2}, k}^{n},
\end{aligned}
$$

which allows us to evaluate the magnetic field at each time step provided the electric field is known. 
Using the same scheme, we obtain the equations on the electric induction $\mathbf{D}$ :

$$
\begin{aligned}
\left(\mathbb{D}^{t} D_{x}\right)_{j, k}^{n+\frac{1}{2}} & =\left[\left(\mathbb{D}^{y} H_{z}\right)_{j, k}^{n+\frac{1}{2}}-\left(\mathbb{D}^{z} H_{y}\right)_{j, k}^{n+\frac{1}{2}}\right]-\left(\mathbb{D}^{y} H_{x}\right)_{j, k}^{n+\frac{1}{2}} \\
\left(\mathbb{D}^{t} D_{y}\right)_{j+\frac{1}{2}, k}^{n+\frac{1}{2}} & =\left(\mathbb{D}^{z} H_{x}\right)_{j+\frac{1}{2}, k}^{n+\frac{1}{2}}, \\
\left(\mathbb{D}^{t} D_{z}\right)_{j, k+\frac{1}{2}}^{n+\frac{1}{2}} & =-\left(\mathbb{D}^{y} H_{x}\right)_{j, k+\frac{1}{2}}^{n+\frac{1}{2}},
\end{aligned}
$$

where we compute $\left.\left(\partial_{y} H_{z}\right)\right|_{j, k+\frac{1}{2}} ^{n+\frac{1}{2}}$ and $\left.\left(\partial_{z} H_{y}\right)\right|_{j, k+\frac{1}{2}} ^{n+\frac{1}{2}}$ with equations (6) and (7).

First, let us treat the semi-discretization in time of the Maxwell-Bloch equations. Let us assume that all the physical values are computed for time $t \leq t_{n}=n \delta t$. We use the Faraday equations (33) to compute $\mathbf{H}^{n+\frac{1}{2}}$ on the whole domain. Thanks to the Bloch equations (3), we get the density matrix $\rho^{n+\frac{1}{2}}$. With the Ampere equations (34), we obtain $\mathbf{D}^{n+1}$.

To proceed, we need to estimate the electric field $\mathbf{E}^{n+1}$ to solve the Faraday equations (33) at time $t_{n+\frac{3}{2}}$. Four steps will be necessary to obtain its value. The electric field is given by the equation

$$
\mathbf{E}^{n+1}=\eta\left(\mathbf{D}^{n+1}-\mathbf{P}^{n+1}\right)
$$

so we have to approximate the polarization $\mathbf{P}$ at time $t_{n+1}$.

The polarization $\mathbf{P}^{n+1}$ could be computed from

$$
\mathbf{P}^{n+1}=\mathcal{N} \operatorname{tr}\left(\mu_{d} \rho^{n+1}\right),
$$

or from the equation

$$
\left(\mathbb{D}^{t} P_{d}\right)^{n+\frac{1}{2}}=\mathcal{N} \operatorname{tr}\left(\mu \rho^{n+\frac{1}{2}}\right)-\frac{l \mathcal{N}}{\hbar} \operatorname{tr}\left(\mu_{d}\left[V^{n+\frac{1}{2}}, \rho^{n+\frac{1}{2}}\right]\right), d \in\{x, y, z\}
$$

As the density matrix is evaluated at times $t_{n+\frac{1}{2}}$, the first equation would yield an implicit scheme, which we wish to avoid. We use the latter equation to compute the polarization term, so $V^{n+\frac{1}{2}}$ is to be estimated. If we use the centered approximation $V^{n+\frac{1}{2}}=\frac{V^{n+1}+V^{n}}{2}$, we would encounter the same problem as with the first FDTD scheme of Sec. 3.1 .

Thus, we use two more steps to obtain the potential $V^{n+\frac{1}{2}}$. In a first step, we compute

$$
\mathbf{P}^{n+\frac{1}{2}}=\mathcal{N} \operatorname{tr}\left(\mu \rho^{n+\frac{1}{2}}\right)
$$




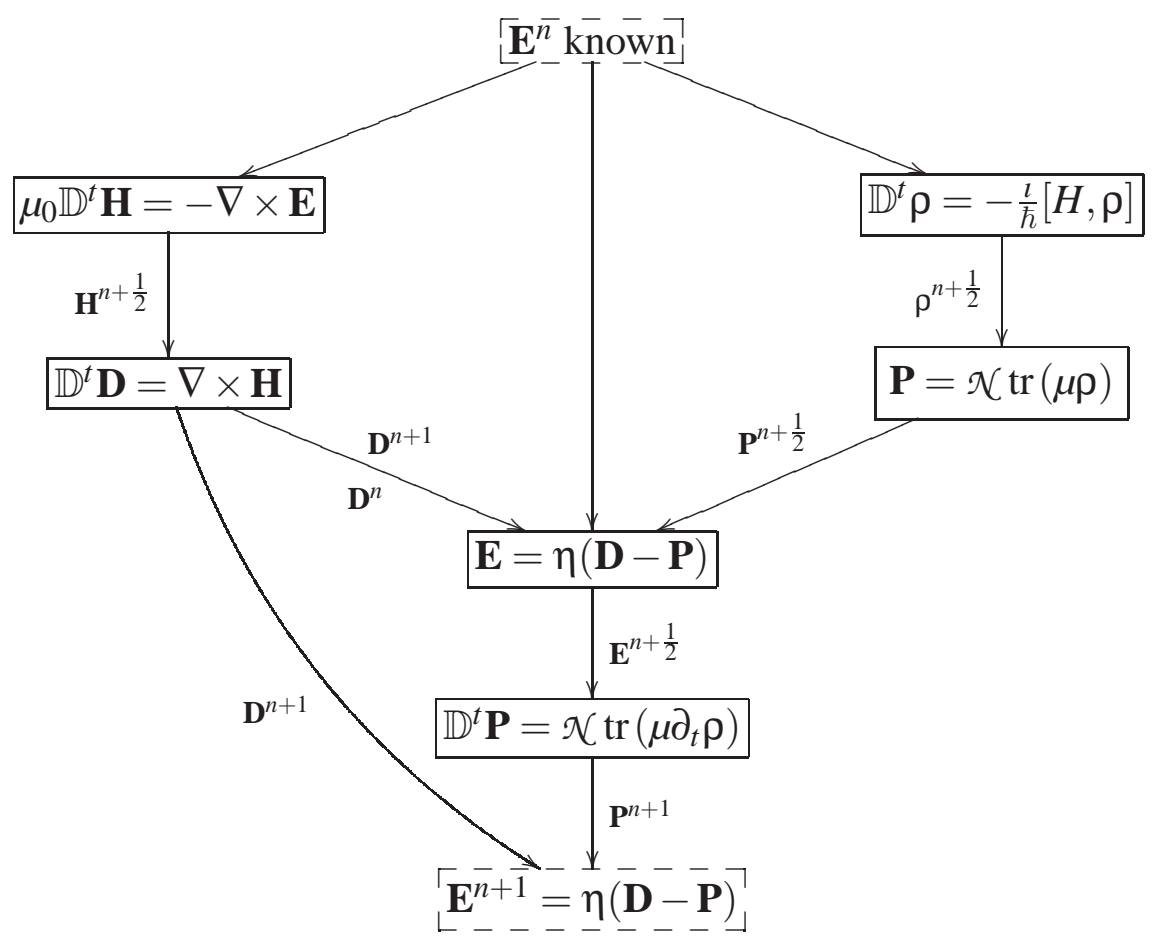

Figure 7. Algorithm of the FDTD scheme with centered nonlinearity.

then the electric field $\mathbf{E}^{n+\frac{1}{2}}$ is obtained through

$$
\mathbf{E}^{n+\frac{1}{2}}=\eta\left(\frac{\mathbf{D}^{n+1}+\mathbf{D}^{n}}{2}-\mathbf{P}^{n+\frac{1}{2}}\right),
$$

It is then straightforward to compute the potential $V^{n+\frac{1}{2}}$ and the polarization $\mathbf{P}^{n+1}$. Finally, we have $\mathbf{E}^{n+1}$ from Eq. (35).

The algorithm is summarized in Fig. 7.

For describing the full discretization of the equations, we introduce a notation: for a function $u$ on the grid, we define the central averaging operator $\mathbb{A}$ by

$$
\left(\mathbb{A}^{y} u\right)_{j, .}=\frac{u_{j+\frac{1}{2}, .}+u_{j-\frac{1}{2}, .}}{2}
$$

using the same idea, we define $\mathbb{A}^{z}, \mathbb{A}^{t}$.

The electric field $\mathbf{E}$ is discretized as $\mathbf{D}$ for times $t_{n}$ and as $\mathbf{P}$ for times $t_{n+\frac{1}{2}}$. 
To compute $\mathbf{E}^{n+\frac{1}{2}}$ from $\mathbf{D}^{n}, \mathbf{D}^{n+1}$ and $\mathbf{P}^{n+\frac{1}{2}}$, we use

$$
\mathbf{E}_{j+\frac{1}{2}, k+\frac{1}{2}}^{n+\frac{1}{2}}=\eta \cdot\left(\begin{array}{c}
\left(\mathbb{A}^{y, z, t} D_{x}\right)_{j+\frac{1}{2}, k+\frac{1}{2}}^{n+\frac{1}{2}}-\left.P_{x}\right|_{j+\frac{1}{2}, k+\frac{1}{2}} ^{n+\frac{1}{2}} \\
\left(\mathbb{A}^{z, t} D_{y}\right)_{j+\frac{1}{2}, k+\frac{1}{2}}^{n+\frac{1}{2}}-\left.P_{y}\right|_{j+\frac{1}{2}, k+\frac{1}{2}} ^{n+\frac{1}{2}} \\
\left(\mathbb{A}^{y, t} D_{z}\right)_{j+\frac{1}{2}, k+\frac{1}{2}}^{n+\frac{1}{2}}-\left.P_{z}\right|_{j+\frac{1}{2}, k+\frac{1}{2}} ^{n+\frac{1}{2}}
\end{array}\right) .
$$

Finally, the electric field $\mathbf{E}^{n+1}$ is obtained from $\mathbf{D}^{n+1}$ and $\mathbf{P}^{n+1}$ through the equations

$$
\begin{aligned}
\left.E_{x}\right|_{j, k} ^{n+1} & =\eta_{x x}\left(\left.D_{x}\right|_{j, k} ^{n+1}-\left(\mathbb{A}^{y, z} P_{x}\right)_{j, k}^{n+1}\right)+\eta_{x z}\left(\left(\mathbb{A}^{z} D_{z}\right)_{j, k}^{n+1}-\left(\mathbb{A}^{y, z} P_{z}\right)_{j, k}^{n+1}\right) \\
\left.E_{y}\right|_{j+\frac{1}{2}, k} ^{n+1} & =\eta_{y y}\left(\left.D_{y}\right|_{J+\frac{1}{2}, k} ^{n+1}-\left.\left(\mathbb{A}^{z} P_{y}\right)\right|_{j+\frac{1}{2}, k} ^{n+1}\right) \\
\left.E_{z}\right|_{j, k+\frac{1}{2}} ^{n+1} & =\eta_{z x}\left(\left(\mathbb{A}^{z} D_{x}\right)_{j, k+\frac{1}{2}}^{n+1}-\left(\mathbb{A}^{y} P_{x}\right)_{j, k+\frac{1}{2}}^{n+1}\right)+\eta_{z z}\left(\left.D_{z}\right|_{j, k+\frac{1}{2}} ^{n+1}-\left(\mathbb{A}^{y} P_{z}\right)_{j, k+\frac{1}{2}}^{n+1}\right) .
\end{aligned}
$$

\subsubsection{Discretization of the Bloch equations}

At the wavelengths, we consider, a KDP crystal is transparent. The relaxation terms in the Bloch equations [16] may be neglected. Thus, the Bloch splitting is no longer necessary as the Bloch equations might be rewritten as

$$
\partial_{t} \rho_{j k}=-\frac{l}{\hbar}\left[H_{0}+V, \rho\right]_{j k},
$$

where $H_{0}=\operatorname{diag}\left(\hbar \omega_{i}\right)_{0 \leq i \leq N}$ is the matrix describing the free Hamiltonian operator.

The Bloch equations can be solved using their analytic solution written at Eq. (15), where we replace $V$ by $V+H_{0}$. The discretization is performed thanks to equations (16) and (17).

\subsubsection{Boundary conditions}

In the direction $z$ of propagation of the wave, we wish to consider the boundaries as transparent for the wave. For this purpose, we shall use the Silver-Müller conditions. Let us recall that these conditions are written in the linear isotropic material (see Fig. 1).

If $\left(\mathbf{D}_{i}, \mathbf{H}_{i}\right)$ is the incident wave-field and $\mathbf{n}$ the outer normal vector to the domain, these conditions are written as

$$
\left(\mathbf{D}-\mathbf{D}_{i}\right) \times \mathbf{n}-\frac{1}{c}\left(\mathbf{H}-\mathbf{H}_{i}\right) \times \mathbf{n} \times \mathbf{n}=0
$$


at the entry of the domain $(z=0)$ and

$$
\mathbf{D} \times \mathbf{n}-\frac{1}{c} \mathbf{D} \times \mathbf{n} \times \mathbf{n}=0,
$$

at the exit.

In the bidimensional case, this yields

$$
\left\{\begin{array}{l}
-D_{y}+D_{y}^{i}+\frac{1}{c}\left(H_{x}-H_{x}^{i}\right)=0 \\
D_{x}-D_{x}^{i}+\frac{1}{c}\left(H_{y}-H_{y}^{i}\right)=0
\end{array}\right.
$$

and

$$
\left\{\begin{array}{l}
D_{y}+\frac{1}{c} H_{x}=0 \\
D_{x}-\frac{1}{c} H_{y}=0 .
\end{array}\right.
$$

These equations are written, using the scheme of Fig. 6, for every $y, z=1, z=N_{z}$ and for time $t_{n+1}$. The missing values of the magnetic field $\mathbf{H}^{n+\frac{3}{2}}$ are obtained with the Faraday equations (33). This gives us the electric induction $\mathbf{D}$ at the boundaries, which could not be recovered from the equations (34). The magnetic field $\mathbf{H}$ is interior to the domain, and can be computed completely from equations (33).

In the transverse direction $y$, we use periodic conditions.

Remark 4 These boundary conditions do not require the entire pulse to be in the domain as an initial datum (as with the PSTD scheme).

\subsubsection{Conclusion}

In this scheme, we have only used central differencing and averaging. The overall accuracy of the scheme is second-order:

Proposition 5 The FDTD scheme with centered nonlinearity is of second order in space and time.

From the dispersion analysis of the FDTD scheme for the Maxwell equations, we can derive the stability criterion of the linear scheme (i.e. when the polarization term is vanishing) [7]

$$
c \delta t \sqrt{\frac{1}{\delta z^{2}}+\frac{1}{\delta y^{2}}}<1,
$$

where $c$ is the speed of light in the medium, $\delta y$ and $\delta z$ the two space steps.

Obtaining a stability for the whole nonlinear scheme is a complex task and is still an open question beyond the scope of this article. 


\section{Experiments}

A particular experiment will be of much importance in this section for highlighting numerical problems as well as for studying the validity of the Maxwell Bloch model. This experiment is a run of second harmonic generation. Second harmonic generation is a physical phenomenon, where as the wave-field at the frequency $\omega$ propagates in the crystal, the optical quadratic nonlinearity creates a wave-field at the frequency $2 \omega$. Each molecule of the crystal may generate a second harmonic with its own phase. In order for this second harmonic to grow, these phases must match. In a uniaxial crystal like the KDP, the angle of incidence can be chosen to make these phases match, this angle is called the phase matching angle. Owing to the expression of the quadratic susceptibility of a KDP crystal, we know that (see page 44 of [11] for instance), if the wave is initially polarized in the $y$-direction, the second and even harmonics will appear in the $x$-direction.

The dipolar matrix for a KDP crystal is given in [1]. We believe that the method exposed in [1] could be extended to treat a large class of crystals. For instance, in [2], we perform several computations with an $\mathrm{AgGaS}_{2}$ crystal.

\subsection{Numerical considerations}

To compare the computational cost of the three schemes, we let a wave-field travel through a KDP crystal.

We measure the computations times for the three schemes for various crystalline lengths. In this experiment, we study the propagation of a (5 fs, $200 \mu \mathrm{m}, 10^{9} \mathrm{~V} / \mathrm{m}$ ) Gaussian pulse at normal incidence. We took $\delta z=10.6 \mathrm{~nm}$ (100 points per wavelength), $\delta y=5.6 \mu \mathrm{m}$ (100 points in the transverse direction), $\delta t=0.013 \mathrm{fs}$.

We put the incoming wave-field as an initial datum in the linear material (see Fig. 1). Thus the crystal starts after $4 \mu \mathrm{m}$ of this medium.

As shown on Fig. 8, the two new schemes presented in this paper are more than two times faster than the scheme of Sec. 3.1 ([4]). The FDTD scheme [4] is clearly too expensive to be used for a physical experiment.

Remark 6 The scheme [4] is heavily optimized for the testing platform (mainly through [14]). This optimization is still a work in progress for the two other schemes (for instance we could improve the efficiency of the FFT algorithm) and we hope to improve the results shown in Fig. 8. 


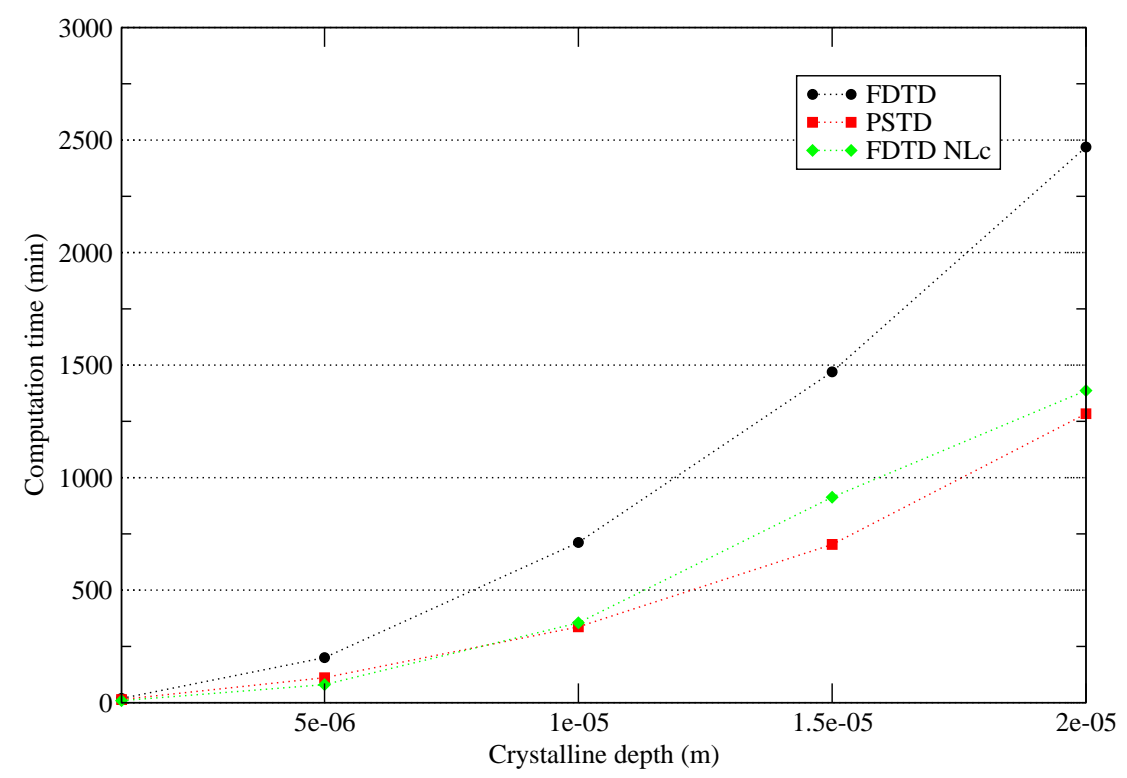

Figure 8. Computation times for the three schemes (PSTD=pseudospectral scheme, NLc=FDTD scheme of Sec. 3.3) for several crystalline lengths on an Intel $^{\circledR}$ $\mathrm{Xeon}^{\mathrm{TM}} 2.2 \mathrm{GHz}$.

\subsubsection{Space and time steps sizes}

In the linear isotropic case, we know from $[3,10]$ that the space step can be chosen as small as 16 points per wavelength (FDTD) or 2 points per wavelength (PSTD). For the one-dimensional Maxwell-Bloch equations in isotropic material, 30 points per wavelength were sufficient [15]. It shall also be noted that this is relative to the wave-length of the higher harmonic we have to study.

In this section, we show that for the nonlinear anisotropic case, space steps have to be taken much smaller than expected. In Fig. 9, we have plotted the results of a second harmonic generation experiment after $20 \mu \mathrm{m}$ of propagation in a crystal of KDP. The incoming wave-field is a $20 \mathrm{fs}$ Gaussian pulse of $10^{8} \mathrm{~V} / \mathrm{m}$ initially polarized in the direction $y$. The even harmonics appear in the $x$-coordinate of the electric field. This coordinate of the field is plotted for several sizes of the space step ranging from 40 to 100 points per wavelength. The $E_{y}$ component does not change with the various step sizes, so we have not plotted it. We have used the pseudospectral scheme for the computations.

From [10], we could expect that a space step of 40 points per wavelength in the fundamental harmonic (that is to say 20 points per wave-length in the second harmonic) would be sufficient to correctly describe the wave-matter interaction. As seen in Fig. 9, this is far from being the case.

Grids coarser than 80 points per wavelength are clearly not adapted to study second harmonic generation and nonlinear effects. 

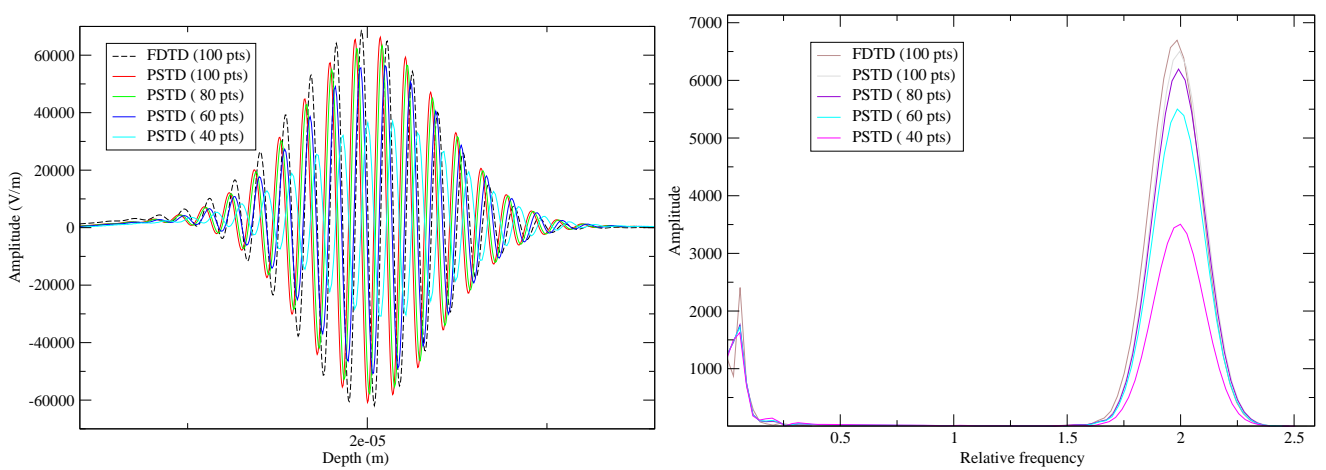

Figure 9. $E_{x}$ component and its Fourier transform in an experiment of second harmonic generation using the pseudospectral (PSTD) scheme for several sizes of the space step. For comparison, we have also plotted the result obtained by the scheme of Sec. 3.1 (FDTD). The frequency is relative to the frequency of the incoming wave-field.

\subsubsection{Bloch splitting}

We could expect that using a Strang splitting method for solving the Bloch equations or not would make a difference only for coarser grids. We run a second harmonic generation experiment at the phase matching angle in $10 \mu \mathrm{m}$ of KDP. The incoming wave is a $20 \mathrm{fs}$ Gaussian pulse of $10^{8} \mathrm{~V} / \mathrm{m}$. The results are plotted on Fig. 10. We have used the one-dimensional PSTD scheme, where the wavelength of the pulse is $1.06 \mu \mathrm{m}$. The time step $\delta t$ is determined from $\delta z$ and the stability criterion of Eq. (31) with a CFL condition of 0.75 .

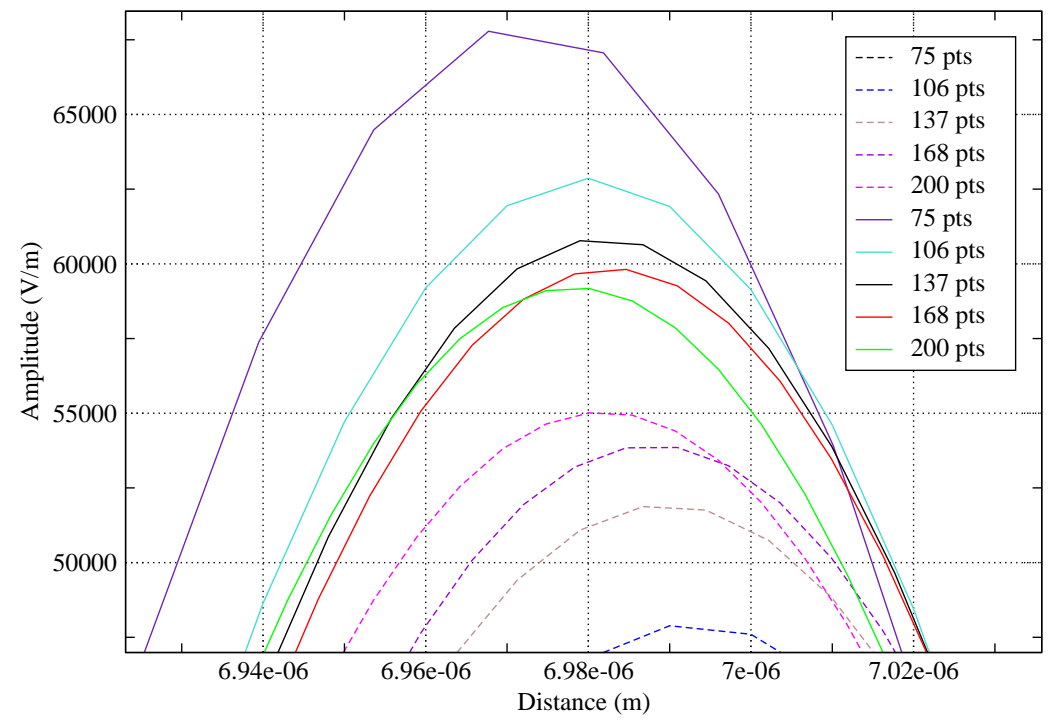

Figure 10. Head of the main peak of the $E_{x}$ component in a second harmonic generation (as in Fig. 9) for several numbers of points per wavelength with a splitting for Bloch equations (dotted lines) or without (plain lines).

Using a splitting method for the Bloch equations, we underestimate the quadratic nonlinearity. As the grid size decreases, the intensity of $E_{x}$ increases. On the contrary, if the Bloch equations are solved directly without a splitting method, we tend 
to overestimate the quadratic nonlinearity. As the grid size decreases, the intensity of $E_{x}$ decreases.

\subsection{Physical experiments}

In the following experiments, instead of plotting the components of the electric field $\mathbf{E}$ of the wave-field as usual, we use the energy flux in each polarization. The energy flux is defined as

$$
\begin{aligned}
& F_{x}(y, z)=\int_{0}^{T} E_{x}(t, y, z) H_{y}(t, y, z) d t \\
& F_{y}(y, z)=\int_{0}^{T} E_{y}(t, y, z) H_{x}(t, y, z) d t
\end{aligned}
$$

where we run the experiment for $t \in[0, T]$.

\subsubsection{Second Harmonic Generation with phase matching}

We will perform a second harmonic generation experiment at the phase matching angle. We inject a Gaussian ( $5 \mathrm{fs}, 200 \mu \mathrm{m}$ wide, $10^{8} \mathrm{~V} / \mathrm{m}$ ) pulse in $10 \mu \mathrm{m}$ of KDP. The pulse is initially polarized in the $y$-direction.

In this run, we used the FDTD scheme of Sec. 3.3. The grid size is 100 points per wavelength in the $z$-direction $(\delta z=10.6 \mathrm{~nm})$. We took 100 points in the direction $y$ and a CFL condition of 0.8 in Eq. (31).

The results are shown in Fig. 11 and 12, where we have plotted two components of the energy flux in the crystal. The coordinate $F_{y}$ contains the fundamental harmonic and odd orders harmonics. The coordinate $F_{x}$ contains even harmonics and in particular the second harmonic.

On Fig. 11, we observe that the fundamental harmonic does not seem to be much affected by the travel through the crystal. The energy at the entry $(y=0 \mu \mathrm{m})$ and at the exit $(\mathrm{y}=10 \mu \mathrm{m})$ is quite the same. Indeed, the crystal length is too short for the SHG process to significantly decrease the intensity of the base harmonic.

On Fig. 12, we observe the evolution of the $F_{x}$ component of the energy flux. We see that initially there is no energy in this polarization. As the pulse propagates, the energy grows. The growth is bigger in the center of the beam, which is where most

intensity of the fundamental harmonic is located. We also observe that the beam is thinner in this polarization.

We will now study the evolution of the electric field at the center of the beam. On 


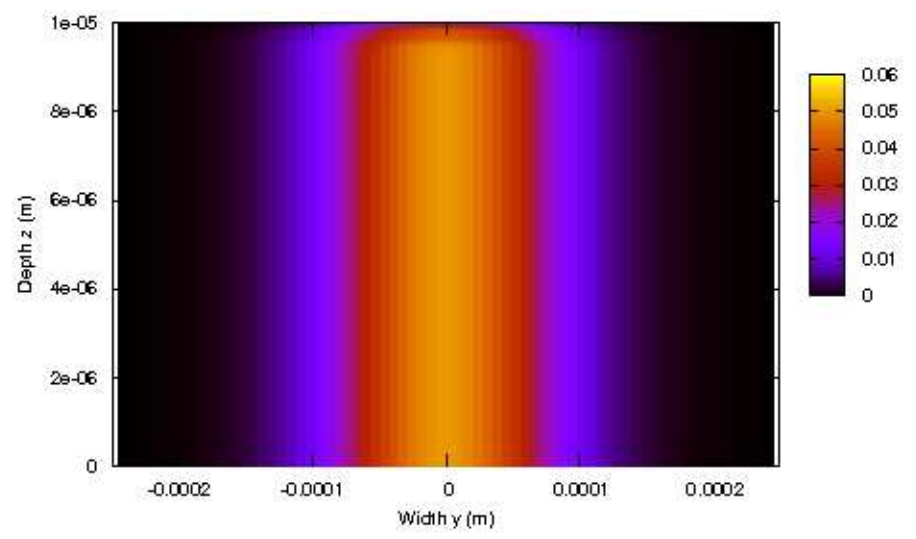

Figure 11. Energy flux $F_{y}$ in a SHG experiment at the phase matching angle after a propagation in $10 \mu \mathrm{m}$ of KDP. We have used arbitrary units for the intensity.

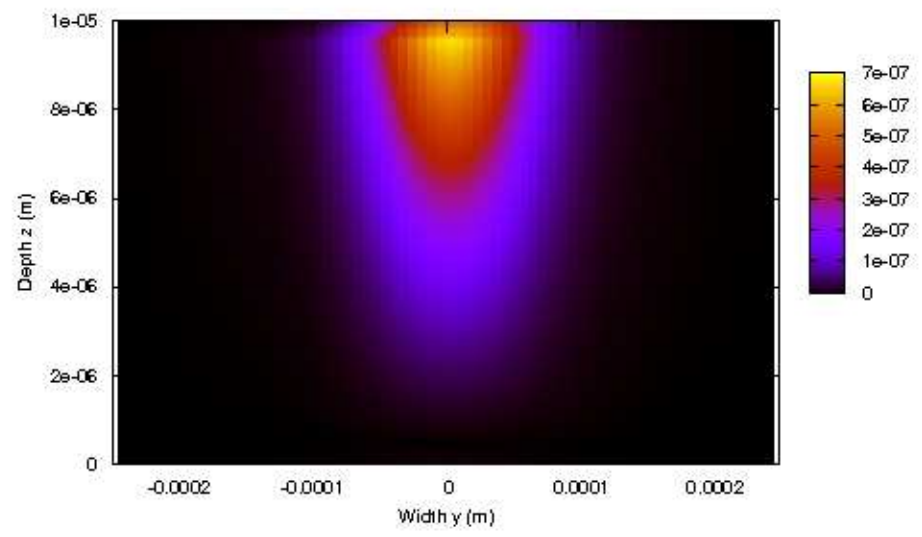

Figure 12. Energy flux $F_{x}$ in a SHG experiment at the phase matching angle after a propagation in $10 \mu \mathrm{m}$ of KDP. We have used arbitrary units for the intensity as in Fig. 11.

Fig. 13, we have plotted the evolution of the coordinate $E_{y}$ of the electric field at seven points in the crystal.

The intensity does not seem much affected by the propagation. The width of the spectrum shall also be noted as we study the propagation of a short pulse. We have plotted the evolution of the $E_{x}$ component on Fig. 14.

We can see the growth of the second harmonic in this polarization. There is also optical rectification as shown on the lower frequencies of the spectrum as expected. 

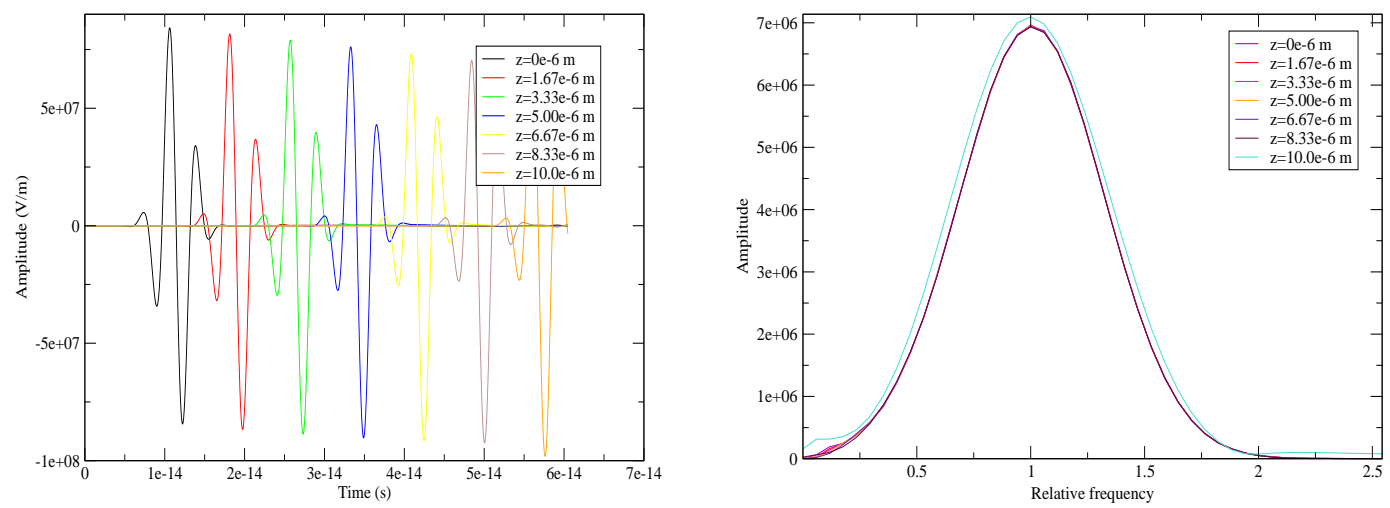

Figure 13. The $E_{y}$ component and its Fourier transform in an experiment of second harmonic generation for seven points in the crystal. The amplitude of the Fourier transform is plotted in arbitrary units, the frequency is relative to the one of the incoming beam.
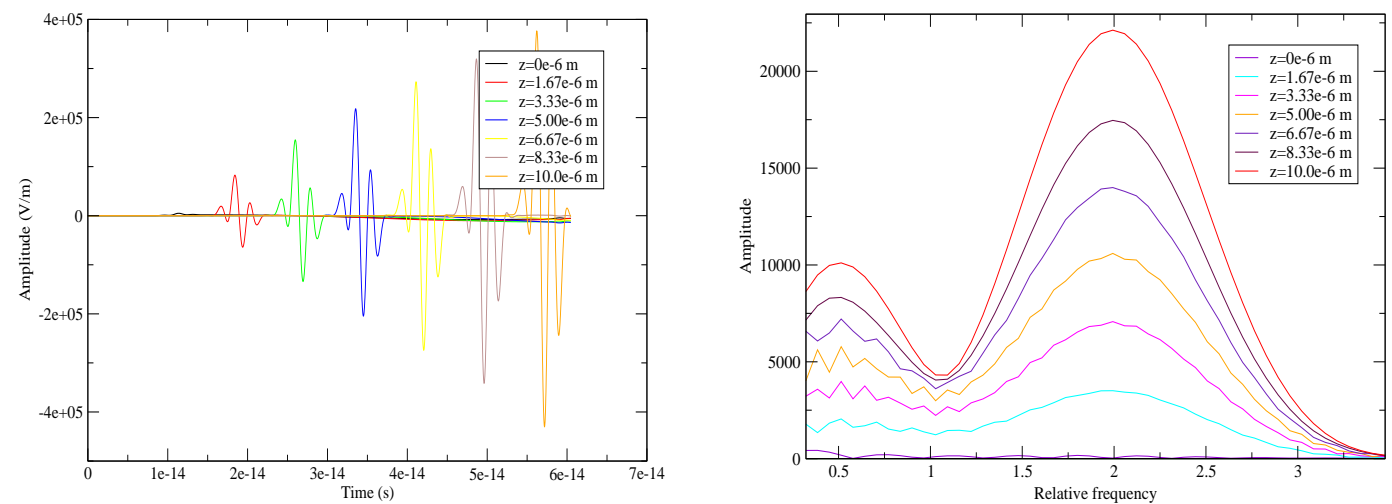

Figure 14. The $E_{x}$ component and its Fourier transform in an experiment of second harmonic generation for seven points in the crystal. The frequency units of the Fourier transform is relative to the frequency of the incoming laser pulse. The amplitude of the spectrum is shown in arbitrary units.

\subsubsection{Second Harmonic Generation without phase matching}

In this section, we study the effect of phase mismatch on the second harmonic growth. In this run, we take an angle of incidence far from the phase matching. We also choose to take a very thin beam in order to have an important diffraction effect.

The diffraction will bend the wavefront of the propagating wave-field. The phase matching condition will then be fulfilled in another direction that the direction of propagation $z$.

In this experiment, the laser beams impinges a KDP crystal of $50 \mu \mathrm{m}$ at normal incidence. We consider a $3 \mathrm{fs}$ Gaussian pulse, whose intensity is $10^{10} \mathrm{~V} / \mathrm{m}$ and width $4 \mu \mathrm{m}$.

We use the pseudospectral scheme and take 80 points per wavelength in the direction of propagation and 150 points in the transverse direction, which gives $\delta z=$ 
$11.8 \mathrm{~nm}, \delta y=149.3 \mathrm{~nm}$ and $\delta t=0.0014 \mathrm{fs}$.

The two components of the energy flux after 400 fs are shown on Fig. 15 and 16.

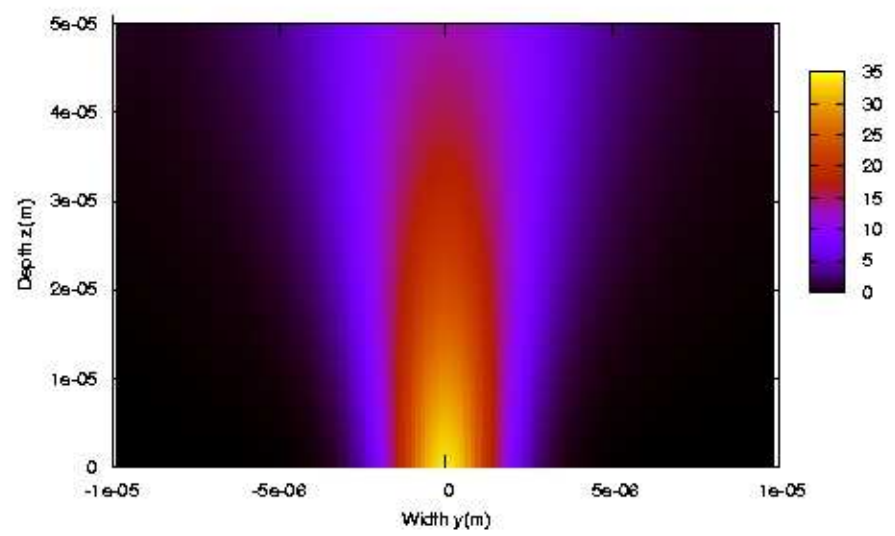

Figure 15. Energy flux $F_{y}$ in a SHG experiment without phase matching after a propagation in $50 \mu \mathrm{m}$ of KDP (268 fs). We have used the same arbitrary units for the intensity.

On Fig. 15, we can observe the effect of the diffraction on the laser beam.

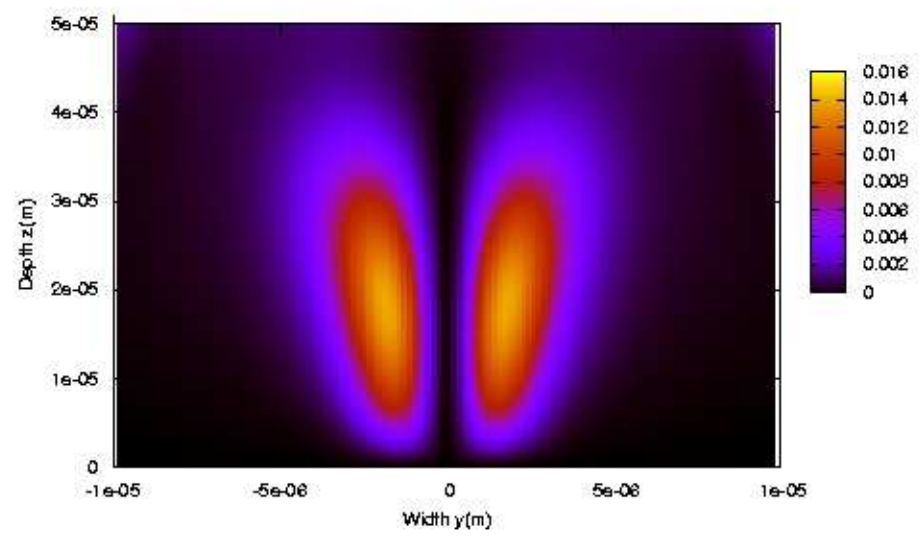

Figure 16. Energy flux $F_{x}$ in a SHG experiment without phase matching after a propagation in $50 \mu \mathrm{m}$ of KDP (268 fs). We have used the same arbitrary units for the intensity as in Fig. 15.

On Fig. 16, second harmonic generation is observable. The two second harmonic pulses do not propagate in the direction of propagation of the fundamental harmonic. After a few microns, the diffraction starts to attenuate the intensity of the laser beam. 


\subsubsection{Self-focusing effect}

For intense laser beams, the optical Kerr effect can produce self-focusing. In this run, we study this effect with our model.

We shall consider a $7.5 \mathrm{fs}$ Gaussian pulse, whose strength is $1.5 \times 10^{10} \mathrm{~V} / \mathrm{m}$ and width $12 \mu \mathrm{m}$. We have taken a very intense laser beam to reduce the crystalline depth needed to observe the self-focusing effect. The wave-field impinges a KDP crystal of $90 \mu \mathrm{m}$ at normal incidence.

We use the pseudospectral scheme of Section 3.2. For this experiment, we took 100 points per wave-length in the direction $z(\delta z=10.6 \mathrm{~nm})$ and 150 points in the transverse direction $y(\delta y=149.3 \mathrm{~nm})$. The CFL parameter is taken to be 0.8 $(\delta t=0.0014 \mathrm{fs}$ ). To avoid the wraparound effect, we have added 5 PML cells in each transverse boundary and 10 in the normal boundaries.

To speed-up the computations, we have used two CPU as described in paragraph 3.2.3.

On Fig. 17, we have plotted the energy flux $F_{y}$ after $400 \mathrm{fs}$.

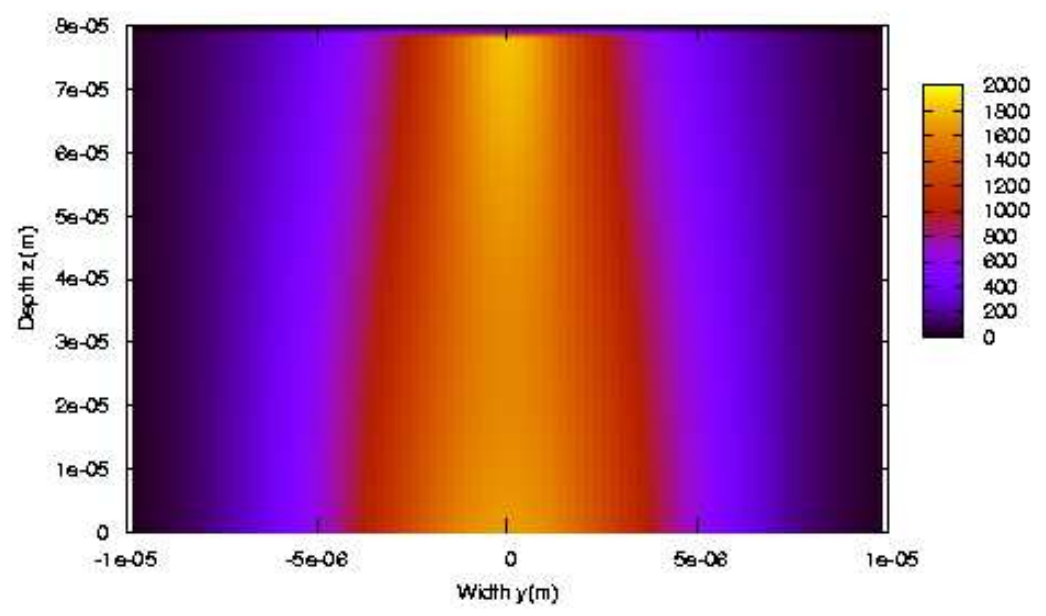

Figure 17. Energy flux after 400 fs of propagation in a KDP crystal. We have used arbitrary units for the intensity of the energy flux.

The self-focusing effect is easily observable on Fig. 17. The Maxwell-Bloch model renders the cubic nonlinearity of the crystal. Yet, we shall note that for such intensity, the crystal would have melt. To study such phenomenon, we should complexify the model to treat the ionization of the material.

However, for practical applications, it is important to have a correct rendering of 
the cubic nonlinearity in nonlinear crystals. Indeed, as shown in [20], the cubic nonlinearity may decrease the efficiency of second harmonic generation.

\section{Conclusion}

In this paper, we have presented three numerical schemes for the bidimensional Maxwell-Bloch equations. The first scheme, which is a direct extension of the unidimensional scheme [9] is too computationally expensive to be really useful for distances longer than a few dozens of microns. The second one uses a pseudospectral method and yields a very simple scheme, which can be easily parallelized. The last FDTD scheme is quite fast to solve compared to the first scheme. It is also rather simple to write as the computation of the nonlinearity is separated from the solving of the Maxwell equations.

For large distances of propagation and short pulses, one should use the PSTD scheme as it is the fastest of the three exposed in this paper. To study longer pulses, the last FDTD scheme is more efficient as the laser beam does not have to be entirely contained in the computational domain as with the pseudospectral scheme.

We have also shown the high complexity of the nonlinearity rendered by the MaxwellBloch model. As shown on paragraph 4.1.1, the grids used have to be much finer than with Schrödinger models for instance. This could be explained by the interplay between the anisotropy of the material and the optical nonlinearities. The MaxwellBloch model renders many physical effects (high-order dispersive nonlinearities, saturation effect, Raman scattering as shown in [2]). Their relative contributions are hard to fully understand.

The schemes presented in this paper could easily be extended to treat three-dimensional wave-fields. However, the amount of computations involved would probably be beyond the reach of common workstations.

\section{References}

[1] C. Besse, B. Bidégaray, A. Bourgeade, P. Degond, O. Saut, A Maxwell-Bloch model with discrete symmetries for wave propagation in nonlinear crystals: an application to KDP, M2AN Math. Model. Numer. Anal. 38 (2) (2004) 321-344.

[2] A. Bourgeade, O. Saut, Comparison between the Maxwell-Bloch and two nonlinear Maxwell models for ultrashort pulses propagation in nonlinear crystals, submitted (2004).

[3] K. S. Yee, Numerical solution of initial boundary value problems involving Maxwell's equations in isotropic media, IEEE Trans. Antennas Propag. AP-14 (1966) 302-307. 
[4] O. Saut, Bidimensional study of the Maxwell-Bloch model in a nonlinear crystal, submitted (2004).

[5] H. Bakker, P. Planken, H. Muller, Numerical calculation of optical frequencyconversion processes : a new approach, J. Opt. Soc. Am. B 6 (9) (1989) 1665-1672.

[6] R. Maleck Rassoul, A. Ivanov, E. extscFreysz, A. Ducasse, F. Hache, Second-harmonic generation under phase-velocity and group-velocit y mismatch: influence of cascading self-phase and cross-phase modulation, Opt. Lett. 22 (5) (1997) $268-270$.

[7] A. Taflove, S. Hagness, Computational electrodynamics: the finite-difference timedomain method, 2nd Edition, Artech House Inc., Boston, MA, 2000.

[8] A. Bourgeade, E. Freysz, Computational modeling of second-harmonic generation by solution of full-wave vector Maxwell equations, J. Opt. Soc. Am. B 17 (2) (2000) 226-234.

[9] O. Saut, Computational modeling of ultrashort powerful laser pulses in an anisotropic crystal, J. Comput. Phys. 197 (2) (2004) 624-646.

[10] Q. Liu, The PSTD algorithm : a time-domain method requiring only two cells per wavelength, Microwave Opt. Techn. Lett. 15 (1997) 158-165.

[11] R. W. Boyd, Nonlinear Optics, Academic Press, 1992.

[12] A. C. Newell, J. V. Moloney, Nonlinear optics, Advanced Topics in the Interdisciplinary Mathematical Sciences, Addison-Wesley Publishing Company Advanced Book Program, Redwood City, CA, 1992.

[13] O. Saut, Étude numérique des nonlinéarités d'un cristal par résolution des équations de Maxwell-Bloch, Ph.D. thesis, INSA Toulouse (2003).

[14] S. Balay, K. Buschelman, W. D. Gropp, D. Kaushik, M. Knepley, L. C. McInnes, B. F. Smith, H. Zhang, PETSc home page, http://www.mcs.anl.gov/petsc (2001).

[15] B. Bidégaray, Time discretizations for Maxwell-Bloch equations, Numer. Methods Partial Differential Equations 19 (3) (2003) 284-300.

[16] B. Bidégaray, A. Bourgeade, D. Reignier, Introducing physical relaxation terms in Bloch equations, J. Comput. Phys. 170 (2) (2001) 603-613.

[17] R. W. Ziolkowski, J. M. Arnold, D. M. Gogny, Ultrafast pulse interactions with twolevel atoms, Phys. Rev. A 52 (4) (1995) 3082-3094.

[18] O. Saut, Une méthode spectrale pour les équations de Maxwell-Bloch bidimensionnelles dans les cristaux non-linéaires, to appear in CRAS Mathématiques (2005).

[19] J.-P. Berenger, A perfectly matched layer for the absorption of electromagnetic waves, J. Comput. Phys. 114 (2) (1994) 185-200.

[20] D. E. T. Ditmire, A.M. Rubenchik, M. Perry, Effects of cubic nonlinearity on frequency doubling of high-power laser pulses, J. Opt. Soc. Am. B 13 (4) (1995) 649655. 